\title{
A classification of phases of bosonic quantum lattice systems in one dimension
}

\author{
Anton Kapustin, Nikita Sopenko, Bowen Yang \\ California Institute of Technology, Pasadena, CA 91125, USA
}

January 14, 2021

\begin{abstract}
We study the entanglement properties of quantum phases of bosonic 1d lattice systems in infinite volume. We show that the ground state of any gapped local Hamiltonian is Short-Range Entangled: it can be disentangled by a fuzzy analog of a finite-depth quantum circuit. We characterize Short-Range Entangled states in terms of decay properties of their Schmidt coefficients. If a Short-Range Entangled state has symmetries, it may be impossible to disentangle it in a way that preserves the symmetries. We show that in the case of a finite unitary symmetry $G$ the only obstruction for the existence of a symmetry-preserving disentangler is an index valued in degree-2 cohomology of $G$. We show that two Short-Range Entangled states are in the same phase if and only if their indices coincide.
\end{abstract}

\section{Introduction}

Gapped phases of lattice systems in 1d are well understood by now [1, 2]. Their classification depends on whether one considers bosonic or fermionic systems, as well as whether other symmetries are present. For example, without imposing any symmetry there is only one bosonic phase (the trivial one). Fermionic phases without symmetries beyond the fermion parity $\mathbb{Z}_{2}^{F}$ are classified by $\mathbb{Z}_{2}$, where the non-trivial element can be realized by the Kitaev chain. Bosonic phases with a unitary on-site symmetry $G$ are classified by the abelian group $H^{2}(G, U(1))$, while fermionic phases with a symmetry $G \times \mathbb{Z}_{2}^{F}$ are classified by $\mathbb{Z}_{2} \times \mathcal{F}$ where $\mathcal{F}$ is an extension of $H^{1}\left(G, \mathbb{Z}_{2}\right)$ by $H^{2}(G, U(1))$. An element of an abelian group describing the phase will be called its index. Most of these classification results have been obtained by assuming that every phase of a $1 \mathrm{~d}$ lattice system can be described by an injective Matrix Product State (MPS).

Although ground states of generic gapped Hamiltonians are not MPS, for finite 1d systems one can find an MPS approximation of any such state. There is an efficient 
numerical procedure (DMRG) for doing this. Thus from a practical standpoint the situation in $1 \mathrm{~d}$ is very satisfactory: given a gapped Hamiltonian, there is an algorithmic procedure for identifying its phase. But from a theoretical standpoint the MPS approach leaves much to be desired. For example, it is not obvious that the index determined through a highly non-unique MPS approximation depends only on the original state. Neither is it obvious that it is an invariant of the phase (i.e. that it depends only on an appropriately defined equivalence class of states).

Recently, Y. Ogata and collaborators $[3,4,5$ developed an approach to the classification of phases of $1 \mathrm{~d}$ systems which does not rely on using an injective MPS. Instead they work with arbitrary $1 \mathrm{~d}$ states satisfying the split property. The split property is the statement that the weak closure of the algebra of observables on any half-line is a Type I von Neumann algebra. In the bosonic case, this is equivalent to saying that the Hilbert space of the system can be written as a tensor product of Hilbert spaces $\mathcal{H}_{-} \otimes \mathcal{H}_{+}$, so that observables localized on the positive (resp. negative) half-line act non-trivially only on $\mathcal{H}_{+}\left(\right.$resp. $\left.\mathcal{H}_{-}\right)$. The split property, while seemingly obvious, typically fails for gapless systems in infinite volume, because von Neumann algebras more exotic than algebras of bounded operators in a Hilbert space show up. Nevertheless, it was shown by T. Matsui [6] that states of $1 \mathrm{~d}$ lattice systems satisfying the area law (and in particular all gapped ground states of local Hamiltonians [7|) satisfy the split property. It is possible in this approach to define an index of a state and show that it is invariant with respect to a natural notion of equivalence (automorphic equivalence). This property ensures that the index is unchanged under continuous deformations of the Hamiltonian which do not close the gap [8].

In this paper we describe a variant of this approach which has several advantages. First, it largely avoids using the theory of von Neumann algebras (although the split property still plays an important role). Instead we use elementary results from quantum information theory. This allows us to make precise the notion that a quantum phase of matter is a pattern of entanglement in the ground state, a point of view expounded in the monograph Ref. [9]. For example, we characterize Short-Range Entangled states (that is, states which are in the trivial phase) in terms of decay properties of their Schmidt coefficients. These decay properties are implied by the finiteness of certain Renýi entanglement entropies. Combining this with the results of Hastings [7, we deduce that ground states of gapped local bosonic Hamiltonians are Short-Range Entangled.

Second, we use a physically motivated definition of a quantum phase of matter, along the lines of Refs. [10, 9]. In Ref. [9] two systems were said to be in the same phase if, after stacking with some unentangled ancilliary systems, they could be connected by a finite-depth quantum circuit. Our definition is the same, except we replace quantum circuits with their fuzzy analogs, locally-generated automorphisms (LGAs) [11]. This notion of a quantum phase, in principle, applies to both gapped and gapless systems. To focus on gapped phases, Refs. [3, 4, 5 impose the split property. In this paper we focus on states which are "invertible" in the sense of A. Kitaev $[12$. By definition, a system is in an invertible state if, when combined with some anscillary system, it can be compltely disentangled by applying an LGA. The anscillary degrees of freedom may be entangled between themselves but not 
with the original system. We show that for states of $1 \mathrm{~d}$ systems invertibility implies the split property, but unlike the latter, it has a clear physical meaning and can be generalized to higher dimensions.

Our definition of an invertible phase makes sense for states of both bosonic and fermionic systems. In this paper we focus on the bosonic case, since the fermionic case is technically more involved. We show that every bosonic $1 \mathrm{~d}$ state in an invertible phase is Short-Range Entangled, i.e. is in the trivial phase. In the case when a finite unitary on-site symmetry $G$ is present, we define an index valued in an abelian group for arbitrary $G$-invariant invertible $1 \mathrm{~d}$ systems and prove that it is a complete invariant of such systems. That is, two $G$-invariant invertible 1d systems are in the same phase if and only if their indices coincide. Our definition of the index is equivalent to that in $[3,4]$ but is formulated in terms of properties of domain walls for symmetries. We also show that a non-trivial index is an obstruction for a system to have a gapped non-degenerate edge which preserves the symmetry $G$.

The content of the paper is as follows. In Section 2 we formulate our definition of phases and invertible phases as suitable equivalence classes of 1d systems. In Section 3 we define the index for invertible states with unitary symmetries and show that entangled pair states realize all possible values of the index. A reader who is mostly interested in the properties of systems without symmetries can skip this section and proceed to Section 4. In Sections 4.1 and 4.2 we study invertible states of $1 \mathrm{~d}$ systems without symmetries. We show that any such state is Short-Range Entangled and characterize Short-Range Entangled states in terms of decay properties of their Schmidt coefficients. This result implies the absence of long-range order in gapped bosonic 1d systems with short-range interactions. We also show that in 1d any Short-Range Entangled state can be disentangled by an LGA without using unentangled ancillas. In Section 4.3 we classify phases of invertible systems with a finite unitary symmetry group. In Appendix A we show that $G$-invariant pure factorized states of $1 \mathrm{~d}$ systems are all in the trivial stable phase according to our definition. In Appendix B we show that the index of a state can be determined provided one has access to a sufficiently large but finite piece of the system. This implies that a non-trivial index is an obstruction for a system to have a gapped non-degenerate edge which preserves the symmetry $G$.

Acknowledgements: We are grateful to Pasha Etingof and Barry Simon for advice. This research was supported in part by the U.S. Department of Energy, Office of Science, Office of High Energy Physics, under Award Number DE-SC0011632. A.K. was also supported by the Simons Investigator Award. B.Y. acknowledges the Caltech mathematics department for a graduate fellowship awarded in fall 2020.

\section{States and phases of 1d lattice systems}

A $1 \mathrm{~d}$ system is defined by its algebra of observables $\mathscr{A}$. In the bosonic case, this is a $C^{*}$-algebra defined as a completion of the $*$-algebra of the form

$$
\mathscr{A}_{\ell}=\underset{M}{\lim _{j \in[-M, M]}} \bigotimes_{j} \mathscr{A}_{j}
$$


where $\mathscr{A}_{j}=\operatorname{End}\left(\mathscr{V}_{j}\right)$ for some finite-dimensional Hilbert space $\mathscr{V}_{j}$. The numbers $d_{j}=\operatorname{dim} \mathscr{V}_{j}$ are not assumed to be bounded, but we assume that $\log d_{j}$ grows at most polynomially with $|j|$. Elements of the algebra $\mathscr{A}$ are called (quasi-local) observables.

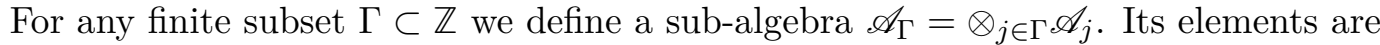
called local observables localized on $\Gamma$. The union of all $\mathscr{A}_{\Gamma}$ is the algebra of local observables $\mathscr{A}_{\ell}$.

There is a distinguished dense $*$-sub-algebra $\mathscr{A}_{a \ell}$ of $\mathscr{A}$ which we call the algebra of almost local observables. $\mathcal{A} \in \mathscr{A}$ is an almost local observable if its commutators with observables in $\mathscr{A}_{j}$ decay faster than any power of $|j|$. More precisely, there exists $k \in \mathbb{Z}$ and a monotonically-decreasing positive (MDP) function $h: \mathbb{R} \rightarrow \mathbb{R}$ which decays faster than any power such that for any $\mathcal{B} \in \mathscr{A}_{j}$ one has $\|[\mathcal{A}, \mathcal{B}]\| \leq\|\mathcal{A}\| \cdot\|\mathcal{B}\| h(|j-k|)$. We will say that $\mathcal{A}$ is $h$-localized on $k$.

Almost local observables are building blocks for Hamiltonians with good locality properties. Such a Hamiltonian is a formal linear combination

$$
F=\sum_{j \in \mathbb{Z}} F_{j},
$$

where $F_{j}$ is a self-adjoint almost local observable which is $h$ localized on $j$ (with the same function $h$ for all $j$ ), and such that the sequence $\left\|F_{j}\right\|, j \in \mathbb{Z}$, is bounded. Note that $F$ is not a well-defined element of $\mathscr{A}$ unless the sum is convergent. To emphasize this, we will call such a formal linear combination a 0-chain rather than a Hamiltonian. Note also that $\operatorname{ad}_{F}(\mathcal{A})=[F, \mathcal{A}]=\sum_{j}\left[F_{j}, \mathcal{A}\right]$ is a well-defined almost local observable for any $\mathcal{A} \in \mathscr{A}_{a \ell}$. It is easy to see that $\operatorname{ad}_{F}$ is an (unbounded) derivation of $\mathscr{A}_{a \ell}$.

The main use of 0-chains is to define a distinguished class of automorphisms of $\mathscr{A}$ which preserve the sub-algebra $\mathscr{A}_{a \ell}$. Consider a self-adjoint 0-chain $F(s)$ which depends continuously ${ }^{1}$ on a parameter $s \in[0,1]$. Then we define a one-parameter family of automorphisms $\alpha_{F}(s), s \in[0,1]$, as a solution of the differential equation

$$
\frac{d}{d s} \alpha_{F}(s)(\mathcal{A})=\alpha_{F}(s)(i[F(s), \mathcal{A}])
$$

with the initial condition $\alpha_{F}(0)=$ Id. One can show that this differential equation has a unique solution for any 0-chain $F(s)$ [13. It follows from Lieb-Robinson bounds that $\alpha_{F}(s)$ maps $\mathscr{A}_{a \ell}$ to itself (see Lemma A.2 of [11]). We will use a short-hand $\alpha_{F}(1)=\alpha_{F}$.

Automorphisms of the form $\alpha_{F}$ for some $F(s)$ will be called locally-generated automorphisms (or LGAs). They are "fuzzy" analogs of finite-depth unitary quantum circuits. Note that locally-generated automorphisms form a group. Indeed, the composition of $\alpha_{F}$ and $\alpha_{G}$ can be generated by the 0-chain

$$
G(s)+\alpha_{G}(s)^{-1}(F(s)) .
$$

The inverse of $\alpha_{F}$ is an automorphism generated by

$$
-\alpha_{F}(s)(F(s)) \text {. }
$$

\footnotetext{
${ }^{1}$ We say that a 0 -chain $F(s)$ depends continuously on $s$ if $F_{j}(s)$ depends continuously on $s$ for all $j \in \mathbb{Z}$.
} 
A state on $\mathscr{A}$ is a positive linear function $\psi: \mathscr{A} \rightarrow \mathbb{C}$ such that $\psi($ Id $)=1$. Given a state $\psi$ and a locally-generated automorphism $\alpha_{F}$, we can define another state by $\alpha_{F}(\psi):=\psi \circ \alpha_{F}(\mathcal{A})=\psi\left(\alpha_{F}(\mathcal{A})\right)$. We will say that $\alpha_{F}(\psi)$ is related by an LGA to $\psi$, or LGA-equivalent to $\psi$.

Following [11], we define a (zero-temperature) phase of a system $\mathscr{A}$ as an LGAequivalence class of pure states on $\mathscr{A}$. We denote by $\Phi(\mathscr{A})$ the set of phases of a $1 \mathrm{~d}$ system $\mathscr{A}$. Our definition of a phase can be motivated as follows. First of all, if $\psi$ is a unique ground states of a gapped almost-local Hamiltonian (that is, the Hamiltonian can be written as a self-adjoint 0-chain), then it is easy to see that any LGA-equivalent state is also a unique ground state of a gapped almost-local Hamiltonian. The converse statement is also true: if two states are unique ground states of gapped Hamiltonians which can be continuously deformed into each other without closing the gap, then the states are related by an LGA 8 . Thus for gapped ground states LGA-equivalence is the same as homotopy equivalence. More generally, it has been proposed to define a zero-temperature phase as an equivalence class of a (not necessarily gapped) pure state under the action of finite-depth quantum circuits $[9]$. One obvious defect of such a definition is that quantum circuits cannot change the range of correlations by more than a finite amount. Thus according to this definition ideal atomic insulators which have a finite correlation range are not in the same phase as any state with exponentially decaying correlations. Replacing finite-depth quantum circuits with LGAs fixes this defect.

A state $\psi$ is called factorized if $\psi(\mathcal{A B})=\psi(\mathcal{A}) \psi(\mathcal{B})$ whenever $\mathcal{A}$ and $\mathcal{B}$ are local observables supported on two different sites. It is easy to see that all factorized pure states of $\mathscr{A}$ are LGA-equivalent. 2 Thus it makes sense to say that the LGAequivalence class of factorized pure states is the trivial phase of $\mathscr{A}$. This is a distinguished element $\tau(\mathscr{A}) \in \Phi(\mathscr{A})$.

To compare states on different $1 \mathrm{~d}$ lattice systems, we define the notion of stable equivalence following A. Kitaev [12]. We say that a pure state $\psi_{1}$ on a system $\mathscr{A}_{1}$ is stably equivalent to a pure state $\psi_{2}$ on a system $\mathscr{A}_{2}$ if there exist systems $\mathscr{A}_{1}^{\prime}$ and $\mathscr{A}_{2}^{\prime}$ and factorized pure states $\psi_{1}^{\prime}$ and $\psi_{2}^{\prime}$ on them such that the pairs $\left(\mathscr{A}_{1} \otimes \mathscr{A}_{1}^{\prime}, \psi_{1} \otimes \psi_{1}^{\prime}\right)$ and $\left(\mathscr{A}_{2} \otimes \mathscr{A}_{2}^{\prime}, \psi_{2} \otimes \psi_{2}^{\prime}\right)$ are LGA-equivalent.

A stable phase is defined to be a stable equivalence class of a pure state $\psi$ on a $1 \mathrm{~d}$ system $\mathscr{A}$. We will denote the set of stable phases by $\Phi$. Any two factorized pure states on any two $1 \mathrm{~d}$ systems are stably equivalent. Thus it makes sense to say that the stable equivalence class of factorized pure states is the trivial stable phase $\tau \in \Phi$. It is also easy to see that the tensor product of systems and their states descends to a commutative associative binary operation $\otimes$ on $\Phi$, and that $\tau$ is the neutral element with respect to this operation. In other words, $(\Phi, \otimes, \tau)$ is a commutative monoid. In the terminology of [9], a system is called Short-Range Entangled if its stable phase is $\tau$.

Next we define what we mean by an invertible stable phase. Following A. Kitaev [12], we will say that a pure state $\psi$ on $\mathscr{A}$ is in an invertible stable phase if there is a $1 \mathrm{~d}$ system $\mathscr{A}^{\prime}$ and a pure state $\psi^{\prime}$ on it such that $\psi \otimes \psi^{\prime}$ is in a trivial stable

\footnotetext{
${ }^{2}$ It is important here that we are dealing with bosonic systems. The statement is not true in the fermionic case.
} 
$1 \mathrm{~d}$ phase. In other words, $x \in \Phi$ is an invertible stable phase if it has an inverse. Therefore invertible stable phases form an abelian group which we denote $\Phi^{*}$.

Note that we defined a phase without any reference to a Hamiltonian. In general, given a pure state, it is not clear whether it is a ground state of any short-range Hamiltonian. Here by a short-range Hamiltonian we mean a Hamiltonian in which interactions between sites decay faster than any power of the distance and thus can be represented by a self-adjoint 0 -chain. However, if a certain phase contains a state which is a ground state of a such a Hamiltonian, then all states in this phase are ground states of short-range Hamiltonians. Indeed, suppose $\psi$ is a ground state for a self-adjoint 0-chain $G$. Since $\mathscr{A}_{a \ell}$ is dense in $\mathscr{A}$ and is in the domain of $\operatorname{ad}_{F}$, this is equivalent to saying that for all $A \in \mathscr{A}_{a \ell}$ one has

$$
\psi\left(\mathcal{A}^{*}[G, \mathcal{A}]\right) \geq 0 .
$$

Then $\psi \circ \alpha_{F}$ is a ground state for the self-adjoint 0-chain $\alpha_{F}(G)$. Moreover, one can show that if $\psi$ is the unique gapped ground state in its GNS representation, then $\psi \circ \alpha_{F}$ is also a unique gapped ground state in its GNS representation. In particular, all states in the trivial stable phase are unique gapped ground states of short-range Hamiltonians.

It will be shown below that all invertible 1d systems are Short-Range Entangled ${ }^{3}$ i.e. $\Phi^{*}$ consists of a single element $\tau$. To get a richer problem, we will study systems and phases with on-site symmetries. Recall that we assumed that each $\mathscr{A}_{j}$ is a matrix algebra, i.e. it has the form $\mathscr{A}_{j}=\operatorname{End}\left(\mathscr{V}_{j}\right)$ for some finite-dimensional vector space $\mathscr{V}_{j}$ (the "on-site Hilbert space"). One says that a group $G$ acts on a system $\mathscr{A}$ by unitary on-site symmetries if one is given a sequence of homomorphisms $R_{j}: G \rightarrow U\left(\mathscr{V}_{j}\right)$, $j \in \mathbb{Z}$, where $U\left(\mathscr{V}_{j}\right)$ is the unitary group of $\mathscr{V}_{j}$. Since each $\mathscr{V}_{j}$ is finite-dimensional, it decomposes as a sum of a finite number of irreducible representations of $G$.

Homomorphisms $R_{j}$ give rise to a $G$-action on $\mathscr{A}, \mathscr{A}_{\ell}$ and $\mathscr{A}_{a \ell}:(g, \mathcal{A}) \mapsto$ $\operatorname{Ad}_{R(g)}(\mathcal{A})=R(g) \mathcal{A} R(g)^{-1}$, where

$$
R(g)=\prod_{j \in \mathbb{Z}} R_{j}(g), \quad g \in G .
$$

The latter is a formal product of unitary local observables $R_{j}(g) \in \mathscr{A}_{j}$ such that the automorphism $\operatorname{Ad}_{R(g)}$ is well-defined. A state $\psi$ on a system $\mathscr{A}$ with an on-site action of $G$ is said to be $G$-invariant if it is invariant under $\operatorname{Ad}_{R(g)}$ for all $g \in G$ : $\psi \circ \operatorname{Ad}_{R(g)}=\psi$ for all $g \in G$.

When defining LGA-equivalence of $G$-invariant states on $\mathscr{A}$, one needs to restrict to those LGAs which are generated by $G$-invariant self-adjoint 0 -chains, i.e. selfadjoint 0 -chains $F(s)=\sum_{j} F_{j}(s)$ such that all observables $F_{j}(s)$ are $G$-invariant. As a result, the automorphism it generates is called $G$-equivariant as it commutes with the action of the group. A $G$-invariant phase for a system $\mathscr{A}$ with an on-site action of $G$ is a $G$-equivariant LGA-equivalence class of $G$-invariant pure states on $\mathscr{A}$. We denote by $\Phi_{G}(\mathscr{A})$ the set of $G$-invariant phases of $\mathscr{A}$ (with some particular $G$-action which is not indicated explicitly).

\footnotetext{
${ }^{3}$ This is not true for fermionic systems:Kitaev chain is a counter-example.
} 
The definition of the trivial $G$-invariant phase of $\mathscr{A}$ is not completely obvious. The most general $G$-invariant factorized pure state on $\mathscr{A}$ is uniquely defined by the condition that when restricted to $\mathscr{A}_{j}$ it takes the form

$$
\psi\left(\mathcal{A}_{j}\right)=\left\langle v_{j}\left|\mathcal{A}_{j}\right| v_{j}\right\rangle, \quad \mathcal{A}_{j} \in \mathscr{A}_{j}
$$

where $v_{j} \in \mathscr{V}_{j}$ is a unit vector which transforms in a one-dimensional representation of $G$. Not every $\mathscr{A}$ admits such states. Even if such states on $\mathscr{A}$ do exist, they need not all belong to the same $G$-invariant phase. For example, consider a system where $\mathscr{V}_{j}$ is the trivial one-dimensional representation of $G=\mathbb{Z}_{2}$ for all $j$ except $j=0$, while $\mathscr{V}_{0}$ is a sum of the trivial and the non-trivial one-dimensional representations with normalized basis vectors $e_{+}$and $e_{-}$. There are two different $\mathbb{Z}_{2}$-invariant factorized pure states corresponding to $v_{0}=e_{+}$and $v_{0}=e_{-}$, and they are clearly not related by a $G$-equivariant LGA. We will define the trivial $G$-invariant phase of $\mathscr{A}$ to be the $G$-equivariant LGA-equivalence class of the states of the form (8) where $v_{j}$ is required to be $G$-invariant. This definition makes sense because for any particular $\mathscr{A}$ all such states are in the same $G$-equivariant LGA-equivalence class. With the exception of Appendix A, by a $G$-invariant factorized pure state we will mean a factorized state defined by (8) where all $v_{j}$ are $G$-invariant.

Next we define the notion of a $G$-invariant stable phase. We say that a $G$-invariant pure state $\psi_{1}$ on a system $\mathscr{A}_{1}$ is $G$-stably equivalent to a pure state $\psi_{2}$ on a system $\mathscr{A}_{2}$ if there exist systems $\mathscr{A}_{1}^{\prime}$ and $\mathscr{A}_{2}^{\prime}$ and $G$-invariant factorized pure states $\psi_{1}^{\prime}$ and $\psi_{2}^{\prime}$ on them such that the pairs $\left(\mathscr{A}_{1} \otimes \mathscr{A}_{1}^{\prime}, \psi_{1} \otimes \psi_{1}^{\prime}\right)$ and $\left(\mathscr{A}_{2} \otimes \mathscr{A}_{2}^{\prime}, \psi_{2} \otimes \psi_{2}^{\prime}\right)$ are related by a $G$-equivariant LGA. A $G$-invariant stable phase is defined to be a $G$-stable equivalence class of a pure $G$-invariant state $\psi$ on a $1 \mathrm{~d}$ system $\mathscr{A}$. We will denote the set of $G$-invariant stable phases by $\Phi_{G}$. $\Phi_{G}$ is a commutative monoid, with the trivial $G$-invariant stable phase as the neutral element $\tau_{G}$. Invertible elements in this monoid form an abelian group which we denote $\Phi_{G}^{*}$. Elements of this group will be called $G$-invertible stable phases.

It is shown in Appendix A that factorized pure states defined by (8) where all $v_{j} \in \mathscr{V}_{j}$ transform in one-dimensional representations of $G$ belong to the trivial $G$-invariant stable phase. This provides a sanity check on our definition of a stable phase: from a physical viewpoint, a nontrivial state must exhibit some entanglement and cannot be factorized.

In this paper we will be studying $G$-invariant stable phases which are also invertible, that is, $G$-invariant stable phases which are mapped to $\Phi^{*}$ by the forgetful homomorphism $\Phi_{G} \rightarrow \Phi$. It is easy to see that every $G$-invertible stable phase is invertible. Less obviously, we will show that every $G$-invariant stable phase which maps to $\Phi^{*}$ is $G$-invertible. We will define an index taking values in an abelian group which classifies such phases. This abelian group is nothing but $\Phi_{G}^{*} \subset \Phi_{G}$. 


\section{An index for invertible 1d systems}

\subsection{Domain wall states}

Consider a $G$-invariant state $\psi$ of $\mathscr{A}$. For any $g \in G$ we let

$$
\rho_{>j}^{g}=\prod_{k>j} \operatorname{Ad}_{R_{k}(g)}
$$

which is a restriction of a locally generated automorphism $\operatorname{Ad}_{R(g)}$ to a half-line $j>0$. Clearly, $\rho_{>j}^{g} \circ \rho_{>j}^{h}=\rho_{>j}^{g h}$. We define the domain wall state $\psi_{>j}^{g}$ by

$$
\psi_{>j}^{g}(\mathcal{A})=\psi\left(\rho_{>j}^{g}(\mathcal{A})\right) .
$$

From now on we suppose that $\psi$ defines a $G$-invariant invertible stable phase. Thus there exists a system $\mathscr{A}^{\prime}$, a factorized pure state $\psi^{\prime}$ on $\mathscr{A}^{\prime}$, and a locallygenerated automorphism of $\mathscr{A} \otimes \mathscr{A}^{\prime}$ that transforms the state $\Psi=\psi \otimes \psi^{\prime}$ to a factorized pure state $\Omega$ on $\mathscr{A} \otimes \mathscr{A}^{\prime}$. We can extend the action of $\rho^{g}$ on $\mathscr{A}$ to an action on $\mathscr{A} \otimes \mathscr{A}^{\prime}$ by making it trivial on $\mathscr{A}^{\prime}$. Then the automorphism

$$
\tilde{\rho}^{g}=\prod_{k \in \mathbb{Z}} \operatorname{Ad}_{\alpha\left(R_{k}(g)\right)}
$$

preserves the state $\Omega$, and its restriction to a half-line $k>j$ is given by $\tilde{\rho}_{>j}^{g}=$ $\alpha^{-1} \circ \rho_{>j}^{g} \circ \alpha$.

We will need the following result from Appendix $\mathrm{C}$ of [11] whose proof we reproduce here for convenience.

Proposition 1. Let $\Omega$ be a factorized pure state on $\mathscr{A}$. Let $\alpha$ be an automorphism of $\mathscr{A}$ which asymptotically preserves $\Omega$, in the sense that there exists a monotonically decreasing positive function $h(r)=O\left(r^{-\infty}\right)$ on $[0, \infty)$ such that for any $\mathcal{A}$ localized on $[r, \infty)$ or $(-\infty,-r]$ one has $|\Omega(\alpha(\mathcal{A}))-\Omega(\mathcal{A})| \leq h(r)\|\mathcal{A}\|$. Then the state $\Omega^{\alpha}: \mathcal{A} \mapsto \Omega(\alpha(\mathcal{A}))$ is unitarily equivalent to $\Omega$.

Proof. Let $\mathscr{A}_{+}$and $\mathscr{A}_{-}$be the $C^{*}$-subalgebras of $\mathscr{A}$ corresponding to $j>0$ and $j \leq 0$, respectively. Let $\Omega_{ \pm}$be the restriction of $\Omega$ to $\mathscr{A}_{ \pm}$, and $\Omega_{ \pm}^{\alpha}$ be the restriction of $\Omega^{\alpha}$ to $\mathscr{A}_{ \pm}$. By Cor. 2.6.11 in [14], the state $\Omega_{+}^{\alpha}$ is quasi-equivalent to $\Omega_{+}$and the state $\Omega_{-}^{\alpha}$ is quasi-equivalent to $\Omega_{-}$. Therefore $\Omega=\Omega_{+} \otimes \Omega_{-}$is quasi-equivalent to $\Omega_{+}^{\alpha} \otimes \Omega_{-}^{\alpha}$. Since both $\Omega$ and $\Omega^{\alpha}$ are pure states, it remains to show that $\Omega^{\alpha}$ is quasi-equivalent to $\Omega_{+}^{\alpha} \otimes \Omega_{-}^{\alpha}$

Let $\rho_{j}, \rho_{j}^{\alpha} \in \mathscr{A}_{j}$ be the density matrices for the restriction of $\Omega$ and $\Omega^{\alpha}$ to $\mathscr{A}_{j}$. The density matrix $\rho_{j}$ is pure, while $\rho_{j}^{\alpha}$ is mixed, in general. But since for any $\mathcal{A} \in \mathscr{A}_{j}$ we have $\left|\operatorname{Tr}\left(\rho_{j}^{\alpha}-\rho_{j}\right) \mathcal{A}\right| \leq h(|j|)\|\mathcal{A}\|$, we have $\left\|\rho_{j}-\rho_{j}^{\alpha}\right\|_{1} \leq h(|j|)$, where $\|\cdot\|_{1}$ is the trace norm. Thus for large $|j|$ the entropy $S_{j}^{\alpha}$ of $\rho_{j}^{\alpha}$ rapidly approaches zero. Specifically, by Fannes inequality, for all sufficiently large $|j|$ we have $S_{j}^{\alpha} \leq h(|j|) \log \left(d_{j} / h(|j|)\right)$. Here $d_{j}^{2}=\operatorname{dim} \mathscr{A}_{j}$. Since we assumed that $\log d_{j}$ grows at most polynomially with $|j|$, $S^{\alpha}=\sum_{j} S_{j}^{\alpha}<\infty$. Therefore the entropy of the restriction of $\Omega^{\alpha}$ to any finite region of $\mathbb{Z}$ is upper-bounded by $S^{\alpha}$. By Theorem 1.5 of $[6]$, the state $\Omega^{\alpha}$ is quasi-equivalent to $\Omega_{+}^{\alpha} \otimes \Omega_{-}^{\alpha}$. 
The automorphism $\rho_{>j}^{g}$ does not preserve $\psi$, but it asymptotically preserves it, by Lemma A.4 of [11]. Therefore $\tilde{\rho}_{>g}^{g}$ asymptotically preserves $\Omega$. Then the above proposition implies that the state $\Omega_{>j}^{g}$ defined by $\Omega_{>j}^{g}(\mathcal{A})=\Omega\left(\tilde{\rho}_{>j}^{g}(\mathcal{A})\right)$ is unitarily equivalent to $\Omega$. Since $\Omega \circ \alpha^{-1}=\Psi$ and $\rho_{>j}^{g}$ acts non-trivially only on $\mathscr{A}$, the states $\psi$ and $\psi_{>j}^{g}$ are also unitarily equivalent.

Moreover, we can identify $\Pi\left(\rho_{>j}^{g}(\mathcal{A})\right)$ with the GNS representation of $\psi_{>j}^{g}$. Specifically, let $\left(\Pi_{>j}^{g}, \mathcal{H}_{>j}^{g},|0\rangle_{>j}^{g}\right)$ be the GNS data of $\psi_{>j}^{g}$ constructed in the usual way. In particular, this coincides with the GNS data $(\Pi, \mathcal{H},|0\rangle)$ of the original state $\psi$ when $g$ is the identity. $\mathcal{H}_{>j}^{g}$ is the completion of $\mathscr{A} / I_{>j}^{g}$ where the left ideal

$$
I_{>j}^{g}:=\left\{\mathcal{A} \mid \psi\left(\rho_{>j}^{g}\left(\mathcal{A}^{*} \mathcal{A}\right)\right)=0\right\} .
$$

Since $\rho_{>j}^{g}\left(I_{>j}^{h}\right)=I_{>j}^{h g^{-1}}, \rho_{>j}^{g}$ induces a linear isometry of Hilbert spaces

$$
\iota_{>j}^{g}: \mathcal{H}_{>j}^{h} \rightarrow \mathcal{H}_{>j}^{h g^{-1}},
$$

such that $\iota_{>j}^{g} \circ \iota_{>j}^{h}=\iota_{>j}^{g h}$ for all $g, h \in G$. Note $\iota$ does not have a fixed source or target. It is a (categorified) group action over all Hilbert spaces $\mathcal{H}_{>j}^{g}$. It then follows that

$$
\iota_{>j}^{g} \Pi_{>j}^{g}(\mathcal{A})\left(\iota_{>j}^{g}\right)^{-1}=\Pi\left(\rho_{>j}^{g}(\mathcal{A})\right) .
$$

This, together with the unitary equivalence proven above, implies that for any $g \in G$ and any $j \in \mathbb{Z}$ there exists a unitary operator $U_{>j}^{g}$ such that

$$
\Pi\left(\rho_{>j}^{g}(\mathcal{A})\right)=U_{>j}^{g} \Pi(\mathcal{A})\left(U_{>j}^{g}\right)^{-1} .
$$

Since the commutant of $\Pi(\mathscr{A})$ consists of scalars, this equation defines $U_{>j}^{g}$ up to a multiple of a complex number with absolute value 1 .

Finally, we are ready to define the index. From equation 140 and $\rho_{>j}^{g} \circ \rho_{>j}^{h}=\rho_{>j}^{g h}$, we infer that

$$
U_{>j}^{g} U_{>j}^{h} \Pi(\mathcal{A})\left(U_{>j}^{g} U_{>j}^{h}\right)^{-1}=U_{>j}^{g h} \Pi(\mathcal{A})\left(U_{>j}^{g h}\right)^{-1} .
$$

Since the commutant of $\Pi(\mathscr{A})$ consists of scalars, it follows that

$$
U_{>j}^{g} U_{>j}^{h}=\nu_{>j}(g, h) U_{>j}^{g h},
$$

where $\nu_{>j}(g, h)$ is a complex number with absolute value 1 . Associativity of operator product implies that $\nu_{>j}(g, h)$ is a 2-cocycle of the group $G$. Its cohomology class is independent of the different choices of $\left\{U_{>j}^{g}: g \in G\right\}$. We claim this cohomology class is an index for bosonic systems. Firstly we show that it is independent of $j$. Then we demonstrate that any $G$-equivariant locally generated automorphism preserves the index. Lastly, we verify that the index of a state in the trivial phase is trivial.

Given $i<j$, we let

$$
R_{(i, j]}^{g}=\prod_{i<k \leq j} R_{k}(g)
$$


By $\sqrt{14}, \Pi\left(R_{(i, j]}^{h}\right)=\Pi\left(\rho_{>j}^{g}\left(R_{(i, j]}^{h}\right)\right)=U_{>j}^{g} \Pi\left(R_{(i, j]}^{h}\right)\left(U_{>j}^{g}\right)^{-1}$, i.e. $\Pi\left(R_{(i, j]}^{h}\right)$ and $U_{>j}^{g}$ commute. Also it is easy to see that

$$
U_{>i}^{g}=\mu(g) \Pi\left(R_{(i, j]}^{g}\right) U_{>j}^{g},
$$

where $\mu(g)$ is a complex number with absolute value 1 . Therefore,

$$
\begin{aligned}
& \nu_{>i}(g, h) \mu(g h) \Pi\left(R_{(i, j]}^{g h}\right) U_{>j}^{g h} \\
= & \nu_{>i}(g, h) U_{>i}^{g h}=U_{>i}^{g} U_{>i}^{h} \\
= & \mu(g) \Pi\left(R_{(i, j]}^{g}\right) U_{>j}^{g} \mu(h) \Pi\left(R_{(i, j]}^{h}\right) U_{>j}^{h} \\
= & \mu(g) \mu(h) \Pi\left(R_{(i, j]}^{g h}\right) U_{>j}^{g} U_{>j}^{h} \\
= & \mu(g) \mu(h) \nu_{>j}(g, h) \Pi\left(R_{(i, j]}^{g h}\right) U_{>j}^{g h} .
\end{aligned}
$$

It is important here that $R_{j}(g)$ is an ordinary (non-projective) representation of $G$. From now on, we fix a domain wall position $j$ as it does not affect the index. We also omit this choice from all notations, for example $\rho_{>j}^{g}$ becomes simply $\rho_{>}^{g}$.

Next, we prove that the index is invariant under an automorphism $\beta:=\beta(1)$ generated by a $G$-equivariant self-adjoint 0 -chain $F(t)=\sum_{j} F_{j}(t)$. Note the site $j$ here is not the domain wall position which has been fixed. In general $\rho_{>}^{g} \circ \beta \neq \beta \circ \rho_{>}^{g}$, however their commutator $\beta \circ \rho_{>}^{g} \circ \beta^{-1} \circ \rho_{>}^{g^{-1}}=\operatorname{Ad}_{\mathcal{B}_{g}}$ for an almost local observable $\mathcal{B}_{g}$. Furthermore,

$$
\mathcal{B}_{g h}=\mathcal{B}_{g} \rho_{>}^{g}\left(\mathcal{B}_{h}\right) .
$$

Before giving the proof, we notice that these facts indeed lead us to the desired invariance of cohomology.

Define a new state

$$
\psi^{\beta}(\mathcal{A}):=\psi(\beta(\mathcal{A})) .
$$

Repeating the argument above for state $\psi^{\beta}$, we get

$$
\Pi\left(\beta\left(\rho_{>}^{g}(\mathcal{A})\right)\right)=W_{>}^{g} \Pi(\beta(\mathcal{A}))\left(W_{>}^{g}\right)^{-1} .
$$

However,

$$
\begin{aligned}
& \Pi\left(\beta\left(\rho_{>}^{g}(\mathcal{A})\right)\right) \\
= & \Pi\left(\beta \circ \rho_{>}^{g} \circ \beta^{-1} \circ \rho_{>}^{g^{-1}}\left(\rho_{>}^{g}(\beta(\mathcal{A}))\right)\right) \\
= & \Pi\left(\mathcal{B}_{g}\right) U_{>}^{g} \Pi(\beta(\mathcal{A}))\left(U_{>}^{g}\right)^{-1} \Pi\left(\mathcal{B}_{g}\right)^{-1} .
\end{aligned}
$$

Therefore,

$$
W_{>}^{g}=\mu(g) \Pi\left(\mathcal{B}_{g}\right) U_{>}^{g},
$$

where $\mu(g)$ is a complex number with norm 1 . And finally,

$$
\begin{aligned}
W_{>}^{g} W_{>}^{h} & =\mu(g) \mu(h) \Pi\left(\mathcal{B}_{g}\right) U_{>}^{g} \Pi\left(\mathcal{B}_{h}\right) U_{>}^{h} \\
& =\mu(g) \mu(h) \Pi\left(\mathcal{B}_{g} \rho_{>}^{g}\left(\mathcal{B}_{h}\right)\right) U_{>}^{g} U_{>}^{h} \\
& =\mu(g) \mu(h) \mu(g h)^{-1} \nu(g, h) W^{g h} .
\end{aligned}
$$


To prove that such $\mathcal{B}_{g}$ exists, notice that the automorphism $\beta(t) \circ \rho_{>}^{g} \circ \beta^{-1}(t) \circ \rho_{>}^{g^{-1}}$ is generated by the 0 -chain $F_{g}(t):=\rho_{>}^{g} \circ \beta(t)\left(\rho_{>}^{g^{-1}}(F(t))-F(t)\right)$, which is almost local. For any almost local self-adjoint $\mathcal{A}(t)$ depending continuously on $t$, we can define an almost local unitary observable $E_{\mathcal{A}}(t)$ satisfying the equation

$$
-i \frac{d}{d t} E_{\mathcal{A}}(t)=E_{\mathcal{A}}(t) \mathcal{A}(t)
$$

When viewed as a 0-chain, the automorphism generated by $\mathcal{A}(t)$ is simply $\operatorname{Ad}_{E_{\mathcal{A}}(t)}$. We let $E_{g}(t):=E_{F_{g}}(t)$, then it follows that $\mathcal{B}_{g}=E_{g}(1)$. To establish identity (20), we need two properties of the observables $E_{\mathcal{A}}(t)$ :

$$
\alpha\left(E_{\mathcal{A}}(t)\right)=E_{\alpha(\mathcal{A})}(t),
$$

and

$$
E_{\mathcal{A}}(t) E_{\mathcal{B}}(t)=E_{\mathcal{C}}(t),
$$

where $\mathcal{C}(t)=\alpha_{\mathcal{B}}^{-1}(t)(\mathcal{A}(t))+\mathcal{B}(t)$. Both are easily checked from equation 26.

Then

$$
E_{g}(t) \cdot \rho_{>}^{g}\left(E_{h}(t)\right)=\rho_{>}^{g}\left(E_{G}(t) \cdot E_{h}(t)\right)=\rho_{>}^{g}\left(E_{X}(t)\right) .
$$

By the first property, $G(t)=\beta(t)\left(\rho_{>}^{g^{-1}}(F(t))-F(t)\right)$. By the second property,

$$
\begin{aligned}
X(t)= & \alpha_{F_{h}}^{-1}(t)(G(t))+F_{h}(t) \\
= & \rho_{>}^{h} \circ \beta(t) \circ \rho_{>}^{h^{-1}} \circ \beta^{-1}(t) \circ \beta(t)\left(\rho_{>}^{g^{-1}}(F(t))-F(t)\right) \\
& +\rho_{>}^{h} \circ \beta(t)\left(\rho_{>}^{h^{-1}}(F(t))-F(t)\right) \\
= & \rho_{>}^{h} \circ \beta(t)\left(\rho_{>}^{(g h)^{-1}}(F(t))-F(t)\right) .
\end{aligned}
$$

Therefore, $\rho_{>}^{g}(X(t))=F_{g h}(t)$, which is what we need.

At last, we verify that the index of the trivial phase is trivial. By the previous result, it suffices to compute the index of a factorized state. Since $\psi\left(\rho_{>}^{g}(\mathcal{A})\right)=\psi(\mathcal{A})$ for any factorized state $\psi$, the GNS representation $\Pi=\Pi^{g}$. Equations (13) and (14) imply

$$
\iota_{>}^{g} \Pi(\mathcal{A})\left(\iota_{>}^{g}\right)^{-1}=\iota_{>}^{g} \Pi_{>}^{g}(\mathcal{A})\left(\iota_{>}^{g}\right)^{-1}=\Pi\left(\rho_{>}^{g}(\mathcal{A})\right)=U_{>}^{g} \Pi(\mathcal{A})\left(U_{>}^{g}\right)^{-1} .
$$

As $\iota_{>}^{g} \iota_{>}^{h}=\iota_{>}^{g h}$, the index is trivial.

Taken together, these results imply that the index of an invertible $G$-invariant state on $\mathscr{A}$ depends only on its $G$-invariant stable phase.

Remark 3.1. Let $\mathscr{A}_{>j}=\otimes_{k>j} \mathscr{A}_{k}$ and $\mathscr{A}_{\leq j}=\otimes_{k \leq j} \mathscr{A}_{k}$. We can define commuting von Neumann algebras acting in $\mathcal{H}_{\psi}$ by letting $\mathcal{M}_{>j}=\Pi_{\psi}\left(\mathscr{A}_{>j}\right)^{\prime \prime}$ and $\mathcal{M}_{\leq j}=$ $\Pi_{\psi}\left(\mathscr{A}_{\leq j}\right)^{\prime \prime}$. These two algebras are each other commutants and generate the whole $B\left(\mathcal{H}_{\psi}\right)$. Then the definition of $U_{>j}^{g}$ implies that $U_{>j}^{g} \in \mathcal{M}_{>j}$ and $U_{<j}^{g} \in \mathcal{M}_{\leq j}$. Then it is easy to see that the index we defined above is the same as defined in [15]. 
The stacking law agrees with the group structure of $H^{2}(G, U(1))$. This is seen by taking the domain wall unitary of the stacked system to be the tensor product of the domain wall unitary operators of the respective subsystems. According to (16), the stacked 2-cocycle is the product of the 2-cocycles of the subsystems.

An important property of the index is that it is locally computable: it can be evaluated approximately given the restriction of the state to any sufficiently large segment of the lattice. This is shown in Appendix B. Local computability of the index implies there can be no $G$-invariant invertible interpolation between a state with a non-trivial index and a $G$-invariant factorized pure state. Put more concisely, a $G$-invariant invertible system with a non-trivial index cannot have a $G$-invariant non-degenerate edge.

\subsection{Examples}

In this section we compute the index of some standard examples of non-trivial invertible states.

In the bosonic case, Matrix Product States furnish examples of invertible states invariant under a (finite) symmetry group $G$. To construct such an example, we pick a projective unitary representation $Q$ of $G$ on a finite-dimensional Hilbert space $\mathscr{W}$. Thus we are given unitary operators $Q(g) \in U(\mathscr{W}), g \in G$, satisfying

$$
Q(g) Q(h)=\nu(g, h) Q(g h),
$$

where $\nu(g, h)$ is a 2-cocycle with values in $U(1)$. We take the local Hilbert space $\mathscr{V}_{j}$ to be $\mathscr{W}_{j} \otimes \mathscr{W}_{j}^{*}$, where $\mathscr{W}_{j}$ is isomorphic to $\mathscr{W}$ for all $j \in \mathbb{Z}$. Then $G$ acts on $\mathscr{V}_{j}$ via $R(g)=Q(g) \otimes Q(g)^{*}$. This is an ordinary (non-projective) action.

To define a $G$-invariant state on $\mathscr{A}$, we first specify it on $\mathscr{A}_{l}$ and then extend "by continuity". We note first that

$$
\mathscr{A}_{\ell}=\otimes_{k \in \mathbb{Z}} \operatorname{End}\left(\mathscr{W}_{k} \otimes \mathscr{W}_{k}^{*}\right)=\otimes_{k \in \mathbb{Z}} \operatorname{End}\left(\mathscr{W}_{k}^{*} \otimes \mathscr{W}_{k+1}\right) .
$$

It is understood here that in both infinite tensor product all but a finite number of elements are identity elements. Thus we can get a $G$-invariant pure state on $\mathscr{A}_{\ell}$ by picking a $G$-invariant vector state on $\operatorname{End}\left(\mathscr{W}_{k}^{*} \otimes \mathscr{W}_{k+1}\right)$ for all $k \in \mathbb{Z}$. If the representation $Q$ is irreducible, there is a unique choice of such a state: the one corresponding to the vector $\frac{1}{\sqrt{d}} 1_{\mathscr{W}} \in \mathscr{W}^{*} \otimes \mathscr{W}$, where $d$ is the dimension of $\mathscr{W}$. In the physics literature such a state on $\mathscr{A}_{\ell}$ is known as an entangled-pair state. Let us denote this state $\psi$.

Note that $\psi$ is in an invertible stable phase. Indeed, any vector state on $\operatorname{End}\left(\mathscr{W}_{k}^{*} \otimes\right.$ $\mathscr{W}_{k}$ ) can be mapped to a factorized vector state by a unitary transformation. The product of these unitary transformations for all $k$ gives us a locally-generated automorphism connecting $\psi$ with a factorized pure state on $\mathscr{A}$. In fact, it is easy to see that the $G$-invariant stable phase of $\psi$ is invertible. The inverse system is obtained by replacing $\mathscr{W}$ with $\mathscr{W}^{*}$ and $Q$ with $Q^{*}$.

To describe the GNS Hilbert space corresponding to $\psi$, let us pick an orthonormal basis $|n\rangle, n=1, \ldots, d$, in $\mathscr{W}$ and denote by $|j, n\rangle,\langle j, n|$ the corresponding orthornormal basis vectors for $\mathscr{W}_{j}$ and $\mathscr{W}_{j}^{*}$. Let us also denote by $\left|1_{j}^{\prime}\right\rangle \in \mathscr{W}_{j}^{*} \otimes \mathscr{W}_{j+1}$ the vector 
$\frac{1}{\sqrt{d}} \sum_{n=1}^{d}\langle j, n|\otimes| j+1, n\rangle$. Then the GNS Hilbert space is the completion of the span of vectors of the form

$$
\left(\bigotimes_{k<J}\left|1_{k}^{\prime}\right\rangle\right) \otimes\left\langle J, n_{J}|\otimes| J+1, m_{J}\right\rangle \otimes \ldots \otimes\left\langle J^{\prime}, n_{J^{\prime}}|\otimes| J^{\prime}+1, m_{J^{\prime}}\right\rangle \otimes\left(\bigotimes_{l>J^{\prime}}\left|1_{l}^{\prime}\right\rangle\right),
$$

where $J, J^{\prime}\left(J \leq J^{\prime}\right)$ are integers.

It is easy to check that the operator $U_{>j}^{g}$ is given (up to a scalar multiple) by

$$
U_{>j}^{g}=\bigotimes_{k \leq j} 1_{k} \otimes Q_{j+1}(g) \otimes \bigotimes_{l>j} R_{l}^{\prime}(g)
$$

where $Q_{j+1}(g) \in \operatorname{End}\left(\mathscr{W}_{j+1}\right)$ is given by $a \mapsto Q(g) a, R_{l}^{\prime}(g) \in \operatorname{End}\left(\mathscr{W}_{l}^{*} \otimes \mathscr{W}_{l+1}\right)$ is given by $a \otimes b \mapsto Q(g)^{*} a \otimes Q(g) b$, and $1_{k} \in \operatorname{End}\left(\mathscr{W}_{k} \otimes \mathscr{W}_{k+1}^{*}\right)$ is the identity operator. This is a well-defined operator because $\left|1_{l}^{\prime}\right\rangle$ is invariant under $R_{l}^{\prime}(g)$. Now, since the operators $R_{l}^{\prime}(g)$ define an ordinary (non-projective) representation of $G$, we see that the index of the state $\psi$ is given precisely by the 2-cocycle $\nu(g, h)$.

\section{A classification of invertible phases of bosonic 1d systems}

\subsection{Preliminaries}

In the following, for a state $\psi$ we denote by $\mathcal{H}_{\psi}$ and $\Pi_{\psi}$ the corresponding GNS Hilbert space and GNS representation. For a region $A$ of the lattice, we denote by $\left.\psi\right|_{A}$ the restriction of a state $\psi$ to $\mathscr{A}_{A}$.

An LGA $\alpha$ generated by $F$ which is $f$-local can be represented by an ordered conjugation with $\vec{\prod}_{j \in \Lambda} e^{i G_{j}}$ for almost local observables $G_{j}$ which are $g$-localized for some $g(r)$ that depends on $f(r)$ only. Indeed, suppose we have an $f$-localized 0 -chain. The automorphism

$$
\alpha_{F_{(-\infty, k+1]}} \circ\left(\alpha_{F_{(-\infty, k]}}\right)^{-1}
$$

is a conjugation by an almost local unitary $e^{i G_{j}}$ which is $g$-localized for some $g(r)$ that depends on $f(r)$ only. Conversely, any such ordered conjugation is a representative of some LGA $\alpha_{F}$. Indeed, if $G_{j}$ is $g$-localized for some $g(r)$, the conjugation with $\vec{\prod}_{j \in \Lambda} \mathcal{U}_{j}(s)$ for $\mathcal{U}_{j}(s)=e^{i s G_{j}}$ gives an LGA for some 0-chain $F$ which is $f$-local for some $f(r)$ that depends on $g(r)$ only.

We define a restriction $G=\left.F\right|_{A}$ of a 0 -chain $F$ to a region $A$ by

$$
G_{j}=\int \prod_{k \in \bar{A}} d V_{k} \operatorname{Ad}_{\prod_{k \in \bar{A}} V_{k}}\left(F_{j}\right)
$$

where the integration is over all on-site unitaries $V_{k} \in \mathscr{A}_{k}$ with Haar measure. Note that $[G, \mathcal{A}]=0$ for any $\mathcal{A} \in \mathscr{A}_{\bar{A}}$, i.e. $G=\left.F\right|_{A}$ is localized on $A$. Therefore 
the restriction of the automorphism $\alpha_{F-\left.F\right|_{A}}$ to the sub-algebra $\mathscr{A}_{\bar{A}}$ is equal to the restriction of $\alpha_{F}$. It is also easy to see that $F-\left.F\right|_{A}$ is almost localized on $\bar{A}$ and thus $\alpha_{F-\left.F\right|_{A}}$ is almost localized on $\bar{A}$.

These properties of restriction have several immediate consequences. Let $A$ and $B$ be a left and a right half-chain, correspondingly. We can represent $F$ as

$$
F=\left.F\right|_{A}+\mathcal{F}_{0}+\left.F\right|_{B}
$$

for some almost local observable $\mathcal{F}_{0}$. That allows us to represent $\alpha_{F}$ as

$$
\alpha_{F}=\alpha_{\left.F\right|_{A}} \circ \alpha_{\left.F\right|_{B}} \circ \operatorname{Ad}_{\mathcal{U}_{0}}
$$

for some almost local unitary observable $\mathcal{U}_{0}$. Similarly, let $A, B$ and $C$ be three regions $(-\infty, j),[j, k]$ and $(k, \infty)$, correspondingly, for some sites $j$ and $k$. Then

$$
\alpha_{F}=\alpha_{\left.F\right|_{A}} \circ \alpha_{\left.F\right|_{C}} \circ \alpha_{\left.F\right|_{B}} \circ \operatorname{Ad}_{\mathcal{U}_{j}} \circ \operatorname{Ad}_{\mathcal{U}_{k}}
$$

for some almost local unitaries $\mathcal{U}_{j}$ and $\mathcal{U}_{k} g$-localized at sites $j$ and $k$, correspondingly. Here $g(r)$ that does not depend on $j$ and $k$.

Here and below we will denote by $\Gamma_{r}$ the interval $[-r, r]$. Let $f: \mathbb{Z}_{+} \rightarrow \mathbb{R}_{+}$ be an MDP function such that $f(r)=O\left(r^{-\infty}\right)$. We will say that two states $\psi$ and $\psi^{\prime}$ are $f$-close on $A \subset \mathbb{Z}$ if for any observable $\mathcal{A}$ localized on $A \cap \bar{\Gamma}_{r}$ we have $\left\|\psi(\mathcal{A})-\psi^{\prime}(\mathcal{A})\right\| \leq\|\mathcal{A}\| f(r)$. Note that since all our algebras of observables are simple, all representations of $\mathscr{A}$ are faithful, and thus $\|\mathcal{A}\|$ can be replaced with $\|\Pi(\mathcal{A})\|$ in any representation $\Pi$ of $\mathscr{A}$.

Lemma 4.1. Let $\psi$ and $\psi^{\prime}$ be two pure states on $\mathscr{A}$ which are $f$-close on $\mathbb{Z}$ for some MDP function $f(r)=O\left(r^{-\infty}\right)$. Then $\psi$ and $\psi^{\prime}$ are unitarily equivalent and one can be produced from the other by a conjugation with $e^{i \mathcal{G}}$ for an almost local self-adjoint observable $\mathcal{G}$ which is g-localized at $j=0$ and bounded $\|\mathcal{G}\| \leq C$ for some $g(r)$ and $C$ which only depend on $f(r)$.

Proof. Unitary equivalence of states $\psi$ and $\psi^{\prime}$ follows from Corollary 2.6.11 of 14. Let $\left(\Pi_{\psi}, \mathcal{H}_{\psi},|\psi\rangle\right)$ be GNS data for $\psi$. The state $\psi^{\prime}$ is a vector state corresponding to $\left|\psi^{\prime}\right\rangle \in \mathcal{H}_{\psi}$. Let $\mathcal{V}_{n}$ be a subspace of $\mathcal{H}_{\psi}$ spanned by vectors which can be produced from $|\psi\rangle$ by an observable localized on $\Gamma_{n}$. Note that $\mathcal{V}_{1} \subset \mathcal{V}_{2} \subset \mathcal{V}_{3} \subset \ldots$

Let $n_{0}$ be such that $f\left(n_{0}\right)<1$. Let us temporarily fix $n \geq n_{0}$ and not indicate it explicitly. The Hilbert space $\mathcal{H}_{\psi}$ is isomorphic to $\mathcal{H}_{\Gamma} \otimes \overline{\mathcal{H}}_{\bar{\Gamma}}$, where the Hilbert spaces $\mathcal{H}_{\bar{\Gamma}}$ and $\mathcal{H}_{\bar{\Gamma}}$ carry representations of $\mathcal{A}_{\Gamma}$ and $\mathcal{A}_{\bar{\Gamma}}$, correspondingly.

Let us show that the vector $\left|\psi^{\prime}\right\rangle \in \mathcal{H}_{\psi}$ is not orthogonal to the subspace $\mathcal{V}$ and estimate the angle between them. The restrictions of vector states $\psi$ and $\psi^{\prime}$ to $\mathcal{A}_{\bar{\Gamma}}$ can be described by density matrices $\rho$ and $\rho^{\prime}$ on $\mathcal{H}_{\bar{\Gamma}}$. We have

$$
\left\|\rho-\rho^{\prime}\right\|_{1} \leq \varepsilon
$$

where $\varepsilon=f(n) \in(0,1)$.

Let

$$
\left|\psi^{\prime}\right\rangle=\sum_{j=1}^{M^{\prime}} \sqrt{\lambda_{i}^{\prime}} \xi_{j}^{\prime} \otimes \eta_{j}^{\prime}
$$




$$
|\psi\rangle=\sum_{i=1}^{M} \sqrt{\lambda_{i}} \xi_{i} \otimes \eta_{i}
$$

be the Schmidt decompositions of $\left|\psi^{\prime}\right\rangle$ and $|\psi\rangle$. By completing the orthonormal vectors $\eta_{i}^{\prime}$ and $\eta_{i}$ to orthonormal bases in $\mathcal{H}_{\Gamma}$, we can write

$$
\begin{aligned}
\left|\psi^{\prime}\right\rangle & =\sum_{j=1}^{N} \sqrt{\lambda_{i}^{\prime}} \xi_{j}^{\prime} \otimes \eta_{j}^{\prime} \\
|\psi\rangle & =\sum_{i=1}^{N} \sqrt{\lambda_{i}} \xi_{i} \otimes \eta_{i}
\end{aligned}
$$

where $\lambda_{i}^{\prime}=0$ for $M^{\prime}<i \leq N, \lambda_{i}=0$ for $M<i \leq N$. The vectors $\xi_{i}^{\prime}, M^{\prime}<i \leq N$, are arbitrary unit vectors in $\mathcal{H}_{\bar{\Gamma}}$ which are orthogonal to $\xi_{i}^{\prime}, i \leq M^{\prime}$, and to each other. Similarly, the vectors $\xi_{i}, M<i \leq N$, are arbitrary unit vectors in $\mathcal{H}_{\bar{\Gamma}}$ which are orthogonal to $\xi_{i}, i \leq M$, and to each other.

Fuchs-van de Graaf inequality implies that for fidelity we have

$$
F\left(\rho^{\prime}, \rho\right):=\left\|\left(\rho^{\prime}\right)^{1 / 2}(\rho)^{1 / 2}\right\|_{1} \geq 1-\frac{\varepsilon}{2} .
$$

Let $U$ be any unitary on $\mathcal{H}_{\bar{\Gamma}}$ such that $\xi_{i}=U \xi_{i}^{\prime}$ for $i=1, \ldots, N$. We can parametrize a unit vector $|\chi\rangle$ in the subspace $\mathcal{V}$ as follows:

$$
|\chi\rangle=\sum_{i=1}^{N} \sqrt{\lambda_{i}} U \xi_{i}^{\prime} \otimes V^{T} \eta_{i}^{\prime}
$$

Here $V^{T} \in U\left(\mathcal{H}_{\Gamma}\right)$ is a unitary operator. Let $\tilde{\rho}=U^{-1} \rho U$. We have

$$
F\left(\rho^{\prime}, \rho\right)=\left\|\left(\rho^{\prime}\right)^{1 / 2}\left(U \tilde{\rho} U^{-1}\right)^{1 / 2}\right\|_{1}=\left\|\left(\rho^{\prime}\right)^{1 / 2} U(\tilde{\rho})^{1 / 2}\right\|_{1} \geq 1-\frac{\varepsilon}{2}
$$

and

$$
\left|\left\langle\chi \mid \psi^{\prime}\right\rangle\right|=\left|\operatorname{Tr}\left(\pi V \pi^{\dagger}\left(\rho^{\prime}\right)^{1 / 2} U(\tilde{\rho})^{1 / 2}\right)\right|
$$

where $\pi: \mathcal{H}_{\Gamma} \rightarrow \mathcal{H}_{\bar{\Gamma}}$ is an isometric embedding defined by $\eta_{i}^{\prime} \rightarrow \xi_{i}^{\prime}, i=1, \ldots, N$. Note that all spectral projections of $\rho^{\prime}$ an $\tilde{\rho}$ belong to $\pi\left(\mathcal{H}_{\Gamma}\right)$, and thus the operator $\left(\rho^{\prime}\right)^{1 / 2} U(\tilde{\rho})^{1 / 2}$ has the form $\pi A \pi^{\dagger}$ for some operator $A$ on $\mathcal{H}_{\Gamma}$. Therefore

$$
\left|\left\langle\chi \mid \psi^{\prime}\right\rangle\right|=\left|\operatorname{Tr}_{\mathcal{H}_{\Gamma}}(V A)\right| .
$$

Because of polar decomposition, for any $N \times N$ matrix $A$ one can choose a unitary $V$ so that $|\operatorname{Tr}(V A)|$ reaches its maximum value $\|A\|_{1}$. Thus we can choose $|\chi\rangle \in \mathcal{V}$ so that

$$
\left|\left\langle\chi \mid \psi^{\prime}\right\rangle\right|=\left\|\left(\rho^{\prime}\right)^{1 / 2} U(\tilde{\rho})^{1 / 2}\right\|_{1} \geq 1-\frac{\varepsilon}{2} .
$$

Since $\varepsilon<1,\left|\psi^{\prime}\right\rangle$ is not orthogonal to the subspace $\mathcal{V}$.

For any $n \geq n_{0}$ let $\left|\chi_{n}\right\rangle$ be the normalized projection of $\psi^{\prime}$ to $\mathcal{V}_{n}$. The estimate (54) implies

$$
\left|\left\langle\chi_{n} \mid \chi_{n+1}\right\rangle\right| \geq 1-\varepsilon_{n}
$$


where $\varepsilon_{n}=f(n)$. Let $\mathcal{U}_{n_{0}}=e^{i \mathcal{G}_{n_{0}}}$ be a unitary localized on $\Gamma_{n_{0}}$ that implements a rotation $|\psi\rangle$ to $\left|\chi_{n_{0}}\right\rangle$ with $\left\|\mathcal{G}_{n_{0}}\right\| \leq \pi$. We can also choose unitary observables $\mathcal{U}_{n}$ for $n \geq n_{0}$ localized on $\Gamma_{n+1}$ and satisfying $\left\|1-\mathcal{U}_{n}\right\| \leq\left(2 \varepsilon_{n}\right)^{1 / 2}$ which implement a rotation $\left|\chi_{n}\right\rangle$ to $\left|\chi_{n+1}\right\rangle$, and which therefore can be written as $\mathcal{U}_{n}=e^{i \mathcal{G}_{n}}$ for an observable $\mathcal{G}_{n}$ local on $\Gamma_{n+1}$ with $\left\|\mathcal{G}_{n}\right\| \leq 2\left(\varepsilon_{n}\right)^{1 / 2}$. The ordered product of all such unitaries over $n \geq n_{0}$ can be written as $\mathcal{U}=e^{i \mathcal{G}}$. By construction, this unitary maps $|\psi\rangle$ to $\left|\psi^{\prime}\right\rangle$. Moreover, since $\left\|\mathcal{G}_{n}\right\| \leq 2(f(n))^{1 / 2}$ for $n \geq n_{0}, \mathcal{G}$ is $g$-localized for some MDP function $g(r)=O\left(r^{-\infty}\right)$ that only depends on $f(r)$, and $\|\mathcal{G}\| \leq \sum_{n=n_{0}}^{\infty} 2(f(n))^{1 / 2}+\pi$, a quantity that also depends only on $f(r)$.

Corollary 4.1.1. Let $\psi$ and $\psi^{\prime}$ be distinct vector states on $\mathscr{A}$ which satisfy the conditions of the above lemma. Let $\psi_{s}, s \in[0,1]$, be a path of vector states corresponding to a normalization of the path of vectors $s|\psi\rangle+(1-s)\left|\psi^{\prime}\right\rangle$. Then there exists a continuous path of self-adjoint almost local observables $\mathcal{G}(s)$ h-localized at $j=0$ such that $\psi_{s}=\alpha_{\mathcal{G}}(s)(\psi)$ and $\|\mathcal{G}(s)\| \leq C$, for some $M D P$ function $h(r)=O\left(r^{-\infty}\right)$ and $C>0$ which only depend on $f$.

Proof. By the above lemma $\left|\psi^{\prime}\right\rangle=\Pi_{\psi}(\mathcal{U})|\psi\rangle$ for some $g$-localized $\mathcal{U}$. Therefore the states $\psi_{s}$ are all $g$-close to a state $\psi$. The vectors $\left|\chi_{n, s}\right\rangle$ depend continuously on $s$, therefore we can choose the unitaries $\mathcal{U}_{n, s}$ and the observables $\mathcal{G}_{n, s}$ so that they are continuous functions of $s$. Let $\tilde{\mathcal{U}}_{n, s}$ be a product $\mathcal{U}_{1, s} \cdots \mathcal{U}_{n, s}$ generated by an almost local observable $\tilde{\mathcal{G}}_{n, s}$. It follows from eq. (4) that $\left\|\tilde{\mathcal{G}}_{n+1, s}-\tilde{\mathcal{G}}_{n, s}\right\| \leq 2\left(\varepsilon_{n}\right)^{1 / 2}$. Therefore, the limit $\mathcal{G}(s)=\lim _{n \rightarrow \infty} \tilde{\mathcal{G}}_{n, s}$ is a continuous function of $s$.

\subsection{A classification of invertible phases without symme- tries}

Short-Range Entangled states are invertible by definition. In this section we show that the converse is also true 4 and thus all invertible phases of bosonic $1 \mathrm{~d}$ systems without symmetries are trivial.

We will say that a pure $1 \mathrm{~d}$ state has bounded entanglement entropy if the entanglement entropies of all intervals $[j, k] \subset \mathbb{Z}$ are uniformly bounded.

Remark 4.1. It was shown by Matsui $[6$ that if $\psi$ has bounded entanglement entropy then it has the split property: the von Neumann algebras $\mathcal{M}_{A}=\Pi_{\psi}\left(\mathscr{A}_{A}\right)^{\prime \prime}$ and $\mathcal{M}_{\bar{A}}=\Pi_{\psi}\left(\mathscr{A}_{\bar{A}}\right)^{\prime \prime}$ are Type I von Neumann algebras. Since they are each other's commutants and generate $B\left(H_{\psi}\right)$, they must be Type I factors. Thus $\mathcal{M}_{A} \simeq B\left(\mathcal{H}_{A}\right)$ for some Hilbert space $\mathcal{H}_{A}$, and the restriction $\left.\psi\right|_{A}$ to a half-line $A$ can be described by a density matrix $\rho_{A}$ on $\mathcal{H}_{A}$. In fact, $[6]$ shows that $\mathcal{H}_{A}$ can be identified with the GNS Hilbert space of one of the Schmidt vector states of $\psi$, which are all unitarily equivalent.

Lemma 4.2. Both SRE 1d states and invertible 1d states have bounded entanglement entropy.

\footnotetext{
${ }^{4}$ This is not true in the case of fermionic systems: Kitaev chain provides a counter-example.
} 
Proof. Suppose we have a state $\psi$ obtained from a factorized pure state $\psi_{0}$ by conjugation with almost local unitaries $\mathcal{U}_{j}$ and $\mathcal{U}_{k}$ which are $g$-localized at sites $j$ and $k$, correspondingly. Since conjugation by $\mathcal{U}_{j, k}$ is an automorphism of $\mathscr{A}$ which is almost localized on $j, k$, for any $\mathcal{A}_{l} \in \mathscr{A}_{l}$ we have

$$
\left|\psi_{0}\left(\mathcal{U}_{j} \mathcal{U}_{k} \mathcal{A}_{l} \mathcal{U}_{k}^{*} \mathcal{U}_{j}^{*}\right)-\psi_{0}\left(\mathcal{A}_{l}\right)\right| \leq(g(|j-l|)+g(|k-l|))\left\|\mathcal{A}_{l}\right\| .
$$

By Fannes inequality [16], the entropy of the site $l$ in the state $\psi$ is bounded by $h(|j-l|)+h(|k-l|)$ for some MDP function $h(r)=O\left(r^{-\infty}\right)$ that depends only on $g(r)$ and the asymptotics of $d_{j}$ for $j \rightarrow \pm \infty$. Therefore such a state has a uniform bound on the entanglement entropy of any interval $[j, k]$. The decomposition eq. (43) then implies that the same is true for any SRE or invertible state.

Let $\psi$ be a possibly mixed state on a half-line $A$ which is $f$-close to a pure factorized state $\psi_{0}$ on $A$. By Corollary 2.6 .11 of [14, $\psi$ is normal in the GNS representation of $\psi_{0}$ and can be described by a density matrix.

We say that a set of (ordered) eigenvalues $\left\{\lambda_{j}\right\}$ has $g(r)$-decay if $\varepsilon(k) \leq g(\log (k))$ for some MDP function $g(r)=O\left(r^{-\infty}\right)$, where $\varepsilon(k)=\sum_{j=k+1}^{\infty} \lambda_{j}$.

Lemma 4.3. Let $\psi$ be a state on a half-line $A$ which is $f$-close to a pure factorized state $\psi_{0}$. Then its density matrix (in the GNS Hilbert space of $\psi_{0}$ ) has eigenvalues with $g(r)$-decay for some $g(r)=O\left(r^{-\infty}\right)$ that depends only on $f(r)$ and the asymptotic behavior of $d_{j}=\operatorname{dim} \mathcal{V}_{j}$ for $j \rightarrow \infty$. Conversely, for any density matrix on a half-line (in the GNS Hilbert space of a pure factorized state $\psi_{0}$ ) whose eigenvalues have $g(r)$-decay there is a state on a half-line which has the same eigenvalues and is $f$-close to $\psi_{0}$. Furthermore, one can choose $f(r)$ so that it depends only on $g(r)$ and the asymptotic behavior of $d_{j}=\operatorname{dim} \mathcal{V}_{j}$ for $j \rightarrow \infty$.

Proof. Suppose $\psi$ is $f$-close to $\psi_{0}$. It can be purified on the whole line (e.g. in a system consisting of the given system on a half-line and its reflected copy on the other half-line) by a state which is $f$-close to a pure factorized state on the whole line. By Lemma 4.1, it can be produced from a pure factorized state on the whole line by a unitary observable $\mathcal{U}$ which is $h$-localized for some $h$ which depends only on $f$. Let $\left|\psi_{0}\right\rangle$ be a GNS vector for the corresponding factorized state $\psi_{0}$. By Lemma A.1 of [11], there is an MDP function $h^{\prime}(r)=O\left(r^{-\infty}\right)$ such that for any $r>0$ there is a unitary observable $\mathcal{U}^{(r)}$ localized on a disk $\Gamma_{r}$ of radius $r$ such that

$$
\| \Pi_{\psi_{0}}(\mathcal{U})\left|\psi_{0}\right\rangle-\Pi_{\psi_{0}}\left(\mathcal{U}^{(r)}\right)\left|\psi_{0}\right\rangle \| \leq h^{\prime}(r) .
$$

On the other hand we have

$$
\| \Pi_{\psi_{0}}(\mathcal{U})\left|\psi_{0}\right\rangle-\Pi_{\psi_{0}}\left(\mathcal{U}^{(r)}\right)\left|\psi_{0}\right\rangle\|\geq\| \rho_{A}-\rho_{A}^{(r)} \|_{1}
$$

where $\rho$ is the density matrix for $\psi$ and $\rho^{(r)}$ is the density matrix for $\Pi_{\psi_{0}}\left(\mathcal{U}^{(r)}\right)\left|\psi_{0}\right\rangle$. The tracial distance between any two density matrices $\rho$ and $\rho^{\prime}$ can be bounded from below in terms of their eigenvalues [17:

$$
\left\|\rho-\rho^{\prime}\right\|_{1} \geq \sum_{j=1}^{\infty}\left|\lambda_{j}(\rho)-\lambda_{j}\left(\rho^{\prime}\right)\right|,
$$


where the eigenvalues $\lambda_{i}$ are ordered in decreasing order. Applying this to $\rho$ and $\rho^{(r)}$ and noting that $\rho^{(r)}$ has rank at most $\operatorname{dim} \mathcal{H}_{A \cap \Gamma_{r}}$, we get

$$
\left\|\rho-\rho^{(r)}\right\|_{1} \geq \varepsilon\left(\operatorname{dim} \mathcal{H}_{A \cap \Gamma_{r}}\right) .
$$

Combining (57), (58) and (60) we get

$$
\varepsilon\left(\operatorname{dim} \mathcal{H}_{A \cap \Gamma_{r}}\right) \leq h^{\prime}(r) .
$$

Since $\operatorname{dim} \mathcal{H}_{A \cap \Gamma_{r}}$ is upper-bounded by $\exp \left(c r^{\alpha}\right)$ for some positive constants $c$ and $\alpha$, we have $\varepsilon(k) \leq g(\log (k))$ for some $g(r)=O\left(r^{-\infty}\right)$ which depends only on $f(r)$, c and $\alpha$.

Conversely, suppose we are given a density matrix on a half-line $A$ with eigenvalues $\lambda_{1} \geq \lambda_{2} \geq \ldots$. We may assume that the dimension of $\mathscr{A}_{A}$ is infinite, since otherwise the statement is obviously true. Pick any pure factorized state $\psi_{0}$ on $A$ and choose a basis in each on-site Hilbert space $\mathcal{V}_{j}, j \in A$, such that for all $j$ the first basis vector gives the state $\left.\psi_{0}\right|_{\mathscr{A}_{j}}$. This gives a lexicographic basis $|n\rangle, n \in \mathbb{N}$, in the GNS Hilbert space of $\psi_{0}$. By our assumption on the growth of dimensions of $d_{j}=\operatorname{dim} \mathcal{V}_{j}$, there are positive constants $c$ and $\alpha$ such that for any $r$ and any $n<e^{c r^{\alpha}}$ the vector state $|n\rangle\langle n|$ coincides with $\psi_{0}$ outside of $\Gamma_{r}$. Therefore the state $\sum_{n=1}^{\infty} \lambda_{n}|n\rangle\langle n|$ is $f$-close to $\psi_{0}$, where $f(r)=g\left(c r^{\alpha}\right)=O\left(r^{-\infty}\right)$.

Lemma 4.4. Let $\psi$ and $\tilde{\psi}$ be pure $1 d$ states on $\mathscr{A}$ which have the split property. Suppose their restrictions to a half-line $A=(-\infty, 0)$ coincide, while their restrictions to $\bar{A}=[0,+\infty)$ are $f$-close to the same pure factorized state $\omega$. Let $\Gamma_{n}=[-n, n]$ and $\psi_{n}$ and $\tilde{\psi}_{n}$ be restrictions of $\psi$ and $\psi$ to $\bar{\Gamma}_{n}$. Then

$$
\left\|\psi_{n}-\tilde{\psi}_{n}\right\| \leq g(n),
$$

where $g(n)=O\left(n^{-\infty}\right)$ is some MDP function which depends only on $f(n)$ and the asymptotics of $d_{j}$ for $j \rightarrow+\infty$.

Proof. Since the states are split, we can desribe them using density matrices on appropriate Hilbert spaces. The decomposition of $\mathbb{Z}$ into the union $(-\infty,-n) \sqcup \Gamma_{n} \sqcup$ $(n,+\infty)$ gives rise to a tensor product decomposition $\mathcal{H}=\mathcal{H}_{n_{\sim}}^{-} \otimes \mathcal{H}_{\Gamma_{n}} \otimes \mathcal{H}_{n}^{+}$. Let $\psi_{n}^{+}$ and $\tilde{\psi}_{n}^{+}$be restrictions of $\psi$ and $\tilde{\psi}$ to $(n,+\infty)$, and $\psi_{n}^{-}$and $\tilde{\psi}_{n}^{-}$be restrictions of $\psi$ and $\tilde{\psi}$ to $(-\infty,-n)$. Let $\rho_{n}^{ \pm}$and $\tilde{\rho}_{n}^{ \pm}$be the corresponding density matrices. Let $\rho_{n}$ be the density matrix of $\psi$ restricted to $(-\infty,-n) \sqcup(n,+\infty)$. Since both $\psi$ and $\tilde{\psi}$ are $f$-close to $\psi_{0}$ when restricted to $(0,+\infty)$, we get

$$
\left\|\rho_{n}^{+}-\tilde{\rho}_{n}^{+}\right\|_{1} \leq 2 f(n) .
$$

Consider now the density matrices $\rho_{n}^{+-}=\rho_{n}^{-} \otimes \rho_{n}^{+}$and $\tilde{\rho}_{n}^{+-}=\tilde{\rho}_{n}^{-} \otimes \tilde{\rho}_{n}^{+}$. Since $\rho_{n}^{-}=\tilde{\rho}_{n}^{-}$, and since trace norm is multiplicative under tensor product, we have

$$
\left\|\rho_{n}^{+-}-\tilde{\rho}_{n}^{+-}\right\|_{1} \leq\left\|\rho_{n}^{+}-\tilde{\rho}_{n}^{+}\right\|_{1} \leq 2 f(n)
$$

On the other hand, Fannes inequality implies that for sufficiently large $n$ the entropy of $\rho_{n}^{+}$and $\tilde{\rho}_{n}^{+}$is upper-bounded by an MDP function $h(n)=O\left(n^{-\infty}\right)$, where 
$h(n)$ depends only on $f(n)$ and the asymptotics of $d_{j}$ for $j \rightarrow+\infty$. Therefore mutual informations $I\left(\rho_{n}^{-}: \rho_{n}^{+}\right)$and $I\left(\tilde{\rho}_{n}^{-}: \tilde{\rho}_{n}^{+}\right)$are also upper-bounded by $h(n)$, and the quantum Pinsker inequality implies

$$
\left\|\rho_{n}^{+-}-\rho_{n}\right\|_{1} \leq \sqrt{2 h(n)}
$$

and similarly for tilded density matrices. Combining this with eq. 644), we get

$$
\left\|\rho_{n}-\tilde{\rho}_{n}\right\|_{1} \leq 2 f(n)+2 \sqrt{2 h(n)} .
$$

Lemma 4.5. Let $\psi$ be a pure $1 d$ state on $\mathscr{A}$ which has the split property. Suppose there is an $R>0$ such that $\psi$ is $f$-close to a pure factorized state $\omega^{+}$on $(R,+\infty)$ and is $f$-close to a pure factorized state $\omega^{-}$on $(-\infty,-R)$. Then it is $g$-close to $\omega^{+} \otimes \omega^{-}$for some MDP function $g(r)=O\left(r^{-\infty}\right)$ which depends only on $f$ and the asymptotics of $d_{j}$ for $j \rightarrow \pm \infty$.

Proof. Since the states are split, we can describe them using density matrices on appropriate Hilbert spaces. The decomposition of $\mathbb{Z}$ into the union $(-\infty,-n) \sqcup \Gamma_{n} \sqcup$ $(n,+\infty)$ gives rise to a tensor product decomposition $\mathcal{H}=\mathcal{H}_{n}^{-} \otimes \mathcal{H}_{\Gamma_{n}} \otimes \mathcal{H}_{n}^{+}$. Let $\psi_{n}^{+}$and $\omega_{n}^{+}$be restrictions of $\psi$ and $\omega^{+}$to $(n,+\infty)$, and $\psi_{n}^{-}$and $\omega_{n}^{-}$be restrictions of $\psi$ and $\omega^{-}$to $(-\infty,-n)$. Let $\rho_{n}^{ \pm}$and $\sigma_{n}^{ \pm}$be the corresponding density matrices. Let $\psi_{n}$ be a restriction of $\psi$ to $(-\infty,-n) \cup(n,+\infty)$ with the corresponding density matrix $\rho_{n}$. For $n>R$ we have

$$
\left\|\rho_{n}^{ \pm}-\sigma_{n}^{ \pm}\right\|_{1} \leq f(n)
$$

Since trace norm is multiplicative under tensor product, we have

$$
\left\|\left(\rho_{n}^{-} \otimes \rho_{n}^{+}\right)-\left(\sigma_{n}^{-} \otimes \sigma_{n}^{+}\right)\right\|_{1} \leq\left\|\rho_{n}^{-}-\sigma_{n}^{-}\right\|_{1}+\left\|\rho_{n}^{+}-\sigma_{n}^{+}\right\|_{1} \leq 2 f(n)
$$

On the other hand, Fannes inequality implies that for sufficiently large $n$ the entropy of $\rho_{n}^{ \pm}$is upper-bounded by MDP function $h(n)=O\left(n^{-\infty}\right)$, where $h(n)$ depends only on $f(n)$ and the asymptotics of $d_{j}$ for $j \rightarrow+\infty$. Therefore mutual informations $I\left(\rho_{n}^{-}: \rho_{n}^{+}\right)$are also upper-bounded by $h(n)$, and the quantum Pinsker inequality implies

$$
\left\|\rho_{n}-\left(\rho^{-} \otimes \rho^{+}\right)\right\|_{1} \leq 2 \sqrt{h(n)} .
$$

Combining this with eq. (68), we get

$$
\left\|\rho_{n}-\left(\sigma_{n}^{-} \otimes \sigma_{n}^{+}\right)\right\|_{1} \leq 2 f(n)+2 \sqrt{h(n)} .
$$

Proposition 2. Let $\psi$ be a pure $1 d$ state which has the split property. It can be related to a pure factorized state on $\mathscr{A}$ by an LGA if and only if there is $g(r)=O\left(r^{-\infty}\right)$ such that for any half-line $A$ the density matrix $\rho_{A}$ corresponding to the restriction $\left.\psi\right|_{A}$ has eigenvalues with $g(r)$-decay. 
Proof. By assumption, the restriction of the state $\psi$ to any half-line $A$ can be described by a density matrix $\rho_{A}$. Suppose there exists a 0 -chain $F$ such that $\psi=\alpha_{F}\left(\psi_{0}\right)$ for some pure factorized state $\psi_{0}$. Let $\tilde{\rho}_{A}$ be the density matrix corresponding to a restriction of the state $\alpha_{F-\left(\left.F\right|_{A}\right)}\left(\psi_{0}\right)$ to $A$. Since the states $\alpha_{F}\left(\psi_{0}\right)$ and $\alpha_{F-\left(\left.F\right|_{A}\right)}\left(\psi_{0}\right)$ coincide on $\bar{A}, \rho$ and $\tilde{\rho}$ have the same set of eigenvalues, and since $\tilde{\rho}$ is $f$-close to $\left.\psi_{0}\right|_{A}$ on $A$ for some function $f(r)=O\left(r^{-\infty}\right)$ independent of $A$, by Lemma 4.3 this set has $g(r)$-decay for some $g(r)=O\left(r^{-\infty}\right)$ which is independent of $A$.

Conversely, suppose for any $A$ the density matrix $\rho_{A}$ has $g(r)$-decay of eigenvalues. By Lemma 4.3 for any $k>0$ we can construct a pure state $\omega^{(k)}$ which coincides with $\psi$ on $(-\infty, k]$ and is $f$-close to the same pure factorized state $\psi_{0}$ on $(k,+\infty]$, with $f$ independent of $k$. Then by Lemma 4.4 and Lemma 4.1 , the states $\omega^{(k)}$ and $\omega^{(k+1)}$ are related by an almost local unitary observable which is $h$-localized on $k$ with $h(r)$ which is independent of $k$. Taking the product of such almost local unitaries over all $k \in \mathbb{N}$, we get an LGA which maps $\omega^{(1)}$ to $\psi$.

Similarly, starting with $\omega^{(1)}$ instead of $\psi$, for any $k \in \mathbb{N}$ we can construct a state $\chi^{(k)}$ which coincides with $\omega^{(1)}$ on $[-k,+\infty)$ and is $f$-close to some fixed pure factorized state on $(-\infty,-k)$, with $f$ independent of $k$. By Lemma 4.4 and Lemma 4.1. states $\chi^{(k)}$ and $\chi^{(k+1)}$ are related by an almost local unitary observable which is $h$-localized on $-k$ with $h(r)$ which is independent of $k$. Taking the product of such almost local unitaries over all $k \in \mathbb{N}$, we get an LGA which maps $\chi^{(1)}$ to $\omega^{(1)}$.

The state $\chi^{(1)}$ is $f$-close to a pure factorized state $\psi_{0}^{+}$on $(1,+\infty)$ and $f$-close to a pure factorized state $\psi_{0}^{-}$on $(-\infty,-1)$. Therefore by Lemma 4.5 it is $f$-close to a pure factorized state $\psi_{0}^{-} \otimes \psi_{0}^{+}$on $(-\infty,-1) \sqcup(1,+\infty)$. Then by Lemma $4.1 \chi^{(1)}$ is related by a conjugation with an almost local unitary to a pure factorized state on the whole line. This proves that $\psi$ is related by an LGA to a pure factorized state on the whole line.

Theorem 1. Any invertible bosonic 1d state $\psi$ is SRE.

Proof. Let $\psi^{\prime}$ be an inverse state for $\psi$, and let $\Psi=\psi \otimes \psi^{\prime}$ be a state of the composite system. By Lemma 4.2 and the results of $[6], \Psi, \psi$, and $\psi^{\prime}$ have the split property. They are also factor states and thus for any half-line $A$ the restrictions $\left.\psi\right|_{A},\left.\psi^{\prime}\right|_{A},\left.\Psi\right|_{A}$ can be described by density matrices $\rho_{A}, \rho_{A}^{\prime}$ and $P_{A}$. By Proposition $2 P_{A}$ has $g(r)$-decay of Schmidt coefficients for some MDP function $g(r)=O\left(r^{-\infty}\right)$ which is independent of $A$, and since $P_{A}=\rho_{A} \otimes \rho_{A}^{\prime}$, the same is true for $\rho_{A}$ and $\rho_{A}^{\prime}$. Then by Proposition 2 the state $\psi$ is SRE.

Remark 4.2. Note that Proposition 2 and Theorem 1 imply that both SRE and invertible states are related to a pure factorized state by an LGA without using ancillas.

Remark 4.3. It was proved by Hastings $[7$ that ground states of gapped local Hamiltonians have bounded Rényi entropy $R_{\alpha}$

$$
R_{\alpha}=\frac{1}{1-\alpha} \log \operatorname{Tr} \rho^{\alpha}
$$


for sufficiently $\operatorname{larg} \AA^{5} \alpha<1$. That guarantees $\varepsilon(k) \leq g(\log (k))$ for some $g(r)=$ $O\left(r^{-\infty}\right)$. In fact, the results of 77 imply that $g(r)$ can be chosen to be the same for all decomposition of $\mathbb{Z}$ into two half-lines. Then Proposition 2 implies that all such ground states are SRE.

\subsection{A classification of invertible phases with symme- tries}

In this section we show that the index defined in Section 3 completely classifies invertible phases of $1 \mathrm{~d}$ bosonic lattice systems with unitary symmetries.

Theorem 2. A G-invariant invertible $1 d$ state $\psi$ is in the trivial stable $G$-invariant phase if and only if it has trivial index.

In order to prove the theorem, we need the following result from representation theory. The proof can be found in various sources such as theorem 4.4 in [19].

Lemma 4.6. Let $V$ be a finite dimensional faithful representation of a finite group $G$. For any irreducible representation $W$ of $G, d(L)=\operatorname{dim}_{\operatorname{Hom}}\left(W, V^{\otimes L}\right)>0$ for large enough $L$. Moreover, $d(L)$ grows exponentially with $L$.

Proof of theorem. Let $V$ be a finite dimensional representation of $\mathrm{G}$ whose subrepresentations contain every irreducible representations of $G$ (including the trivial representation). Such $V$ always exists with $\mathbb{C}[G]$ being one example. Clearly $V$ is faithful. For all $j \in \mathbb{Z}$, let $\mathcal{V}_{j}^{\prime}=V$ for all $j \in \mathbb{Z}$. Let $\mathscr{A}_{j}^{\prime}=\operatorname{End}\left(\mathcal{V}_{j}^{\prime}\right)$ and $\psi^{\prime}$ be a factorized $G$-invariant pure state on $\mathscr{A}^{\prime}=\otimes_{j} \mathscr{A}_{j}^{\prime}$ (it exists because $\mathcal{V}_{j}^{\prime}$ contains the trivial representation for all $j$ ). We will now show that the state $\Psi=\psi \otimes \psi^{\prime}$ on $\mathscr{A} \otimes \mathscr{A}^{\prime}$ can be disentangled by a $G$-equivariant LGA.

By Lemma 4.2 and [6], the state $\psi$ has the split property. Thus the von Neumann algebra $\mathcal{M}_{>k}=\Pi_{\psi}\left(\mathscr{A}_{>k}\right)^{\prime \prime}$ on a half-line $(k, \infty)$ is a Type I factor. In other words, $\mathcal{M}_{>k}$ is isomorphic to the algebra of bounded operators on a Hilbert space $\mathcal{W}_{>k}$. By Remark 3.1 and triviality of the index, the operators $U_{>k}^{g}$ define a unitary representation of $G$ on $\mathcal{W}_{>k}$. The split property also implies that $\psi$ restricted to $(k, \infty)$ is a normal state with a density matrix $\rho_{k}$ (a positive operator on $\mathcal{W}_{>k}$ with unit trace). As the restriction of $\psi$ to $\mathscr{A}_{>k}$ is $G$-invariant, each eigenspace of $\rho_{k}$ is a representation of $G$. Therefore, each summand in the direct sum decomposition of $\mathcal{W}_{>k}$ into irreducible (necessarily finite dimensional) representations of $G$ is spanned by eigenvectors of $\rho_{k}$ with equal eigenvalues.

By Lemma 4.6 each of these irreducible summands is contained in $V^{\otimes L}$ for large enough $L$ with exponentially growing multiplicity (with respect to $L$ ). Therefore in the same way as in Lemma 4.3 we can construct a $G$-invariant state on $\mathscr{A}_{>k} \otimes \mathscr{A}_{>k}^{\prime}$ which is $f$-close to a $G$-invariant pure factorized state, has the same eigenvalues as $\rho_{k}$, and the eigenspace for each eigenvalue transforms in the same representation of $G$ as the corresponding eigenspace of $\rho_{k}$. Furthermore, $f(r)=O\left(r^{-\infty}\right)$ can be chosen to be independent of $k$. Therefore there is a $G$-invariant pure state $\Omega^{(k)}$ on

\footnotetext{
${ }^{5}$ In fact it was shown in 18 that it is bounded for any $1>\alpha>0$.
} 
$\mathscr{A} \otimes \mathscr{A}^{\prime}$ that coincides with $\Psi=\psi \otimes \psi^{\prime}$ on $(-\infty, k]$ and is $f$-close to a $G$-invariant pure factorized state on $(k,+\infty)$.

By Proposition 2 there is an LGA $\alpha_{F}$ such that $\alpha_{F}(\Psi)$ is factorized and $G$ invariant. Since $\alpha_{\left.F\right|_{(-\infty, k]}}\left(\Omega^{(k)}\right)$ is $f$-close to a factorized state on $\mathbb{Z}$, by Lemma 4.1 the state $\Omega^{(k+1)}$ can be produced from $\Omega^{(k)}$ by a conjugation with an almost local unitary. Let $\left|\Omega^{(k+1)}\right\rangle$ and $\left|\Omega^{(k)}\right\rangle$ be the corresponding vectors for states $\Omega^{(k)}$ and $\Omega^{(k+1)}$ in $\mathcal{H}_{\Omega^{(k)}}$. Since these vectors are $G$-invariant, their linear combinations are also $G$-invariant and give states $f$-close to a $G$-invariant pure factorized state on $(k, \infty)$. Let $\Omega_{s}$ for $s \in[0,1]$ be the path of of vector states corresponding to the normalization of the path of vectors $s\left|\Omega^{(k+1)}\right\rangle+(1-s)\left|\Omega^{(k)}\right\rangle$. By Corollary 4.1.1 there is an LGA $\alpha_{P}$ generated by an almost local observable $P(s)$, such that $\Omega_{s}=\alpha_{P}(s)\left(\Omega^{(k)}\right)$. Let $P^{G}(s)$ be the observable obtained from $P(s)$ by averaging over the group action. Then $P^{G}(s)$ generates an automorphism $\alpha_{P^{G}}$ which is $G$-invariant, and since the state $\Omega_{s}$ is $G$-invariant for all $s$, we still have $\Omega_{s}=\alpha_{P G}(s)\left(\Omega^{(k)}\right)$. The automorphism $\alpha_{P G}$ is a conjugation by some $G$-invariant almost local unitary $e^{i \mathcal{G}_{k}}$. Taking their ordered product over all $k$, we obtain a $G$-invariant LGA disentangling $\Psi$. Thus $(\mathscr{A}, \psi)$ is in a trivial $G$-invariant phase.

Corollary 2.1. The index defines a group isomorphism between $\Phi_{G}^{*}$ and $H^{2}(G, U(1))$.

Proof. In section 3 we have shown that the index defines a group homomorphism from $\Phi_{G}^{*}$ to $H^{2}(G, U(1))$. The MPS construction in section 3 implies that the homomorphism is surjective. Moreover, the theorem shows that this homomorphism has trivial kernel. Therefore, it defines a group isomorphism.

Corollary 2.2. Every G-invariant invertible system is G-invertible.

Proof. Let $(\mathscr{A}, \psi)$ be a $G$-invariant invertible system. Construct another $G$-invariant system $\left(\mathscr{A}^{\prime}, \psi^{\prime}\right)$ whose index is the group inverse to that of $(\mathscr{A}, \psi)$. This can be done using to the Entangled Pair State construction in section 3. The stacked system $\left(\mathscr{A} \otimes \mathscr{A}^{\prime}, \psi \otimes \psi^{\prime}\right)$ has trivial index according to the stacking rule shown in section 3. The theorem implies that this stacked system is in the trivial stable $G$-invariant phase. Hence, $(\mathscr{A}, \psi)$ is $G$-invertible with an inverse $\left(\mathscr{A}^{\prime}, \psi^{\prime}\right)$.

\section{A Stable equivalence of $G$-invariant factorized pure states}

In this section we study $G$-invariant factorized pure states defined by $(8)$ where the vectors $v_{j}$ may transform in non-trivial one-dimensional representations of $G$. We will show that all such states are in the trivial $G$-invariant stable phase.

Consider first a $1 \mathrm{~d}$ system where for $j \neq 0 \mathscr{V}_{j}=\mathbb{C}$ is the trivial representation of $G$, while $\mathscr{V}_{0}=\mathscr{W}$ is a finite-dimensional representation containing a unit vector $w$ transforming in a non-trivial one-dimensional representation of $G$. Consider a $G$-invariant factorized pure state where $v_{0}=w$ (all other $v_{j}$ are unique up to a scalar multiple). Physically, this corresponds to a non-trivial $G$-invariant ground state of 
a 0 d system regarded as a $G$-invariant state of a $1 \mathrm{~d}$ system. We are going to show that this $1 \mathrm{~d}$ state is in a trivial $G$-invariant stable phase.

Without a loss of generality we may assume that $\mathscr{W}$ contains a $G$-invariant vector $w^{\prime}$. Indeed, if this is not the case, we can tensor the above system with a similar system where $\mathscr{W}$ is replaced with $\mathscr{W}^{*} \oplus \mathbb{C} \cdot e$, where $G$ acts on the second summand by the trivial representation, and $v_{0}=e$. The auxiliary system is in the trivial $G$-invariant phase, so this does not affect the $G$-invariant stable phase of the system we are interested in. Then the composite system has $\mathscr{V}_{0}=\mathscr{W} \otimes \mathscr{W}^{*} \oplus \mathscr{W} \otimes \mathbb{C} \cdot e$, with a $G$-invariant factorized pure state corresponding to $v_{0}$ in the second summand. The first summand now contains a $G$-invariant vector $w \otimes w^{*}$.

Consider now an auxiliary system $\mathscr{A}^{\prime}$ where $\mathscr{V}_{j}^{\prime}=\mathbb{C}$ is the trivial representation for $j \leq 0$ and $\mathscr{V}_{j}^{\prime}=\mathscr{W}^{*} \otimes \mathscr{W}$ for $j>0$. The algebra of local observables $\mathscr{A} \otimes \mathscr{A}^{\prime}$ has the form

$$
\operatorname{End}(\mathscr{W}) \otimes \operatorname{End}\left(\mathscr{W}^{*} \otimes \mathscr{W}\right) \otimes \operatorname{End}\left(\mathscr{W}^{*} \otimes \mathscr{W}\right) \otimes \ldots
$$

We pick a $G$-invariant factorized pure state $\psi^{\prime}$ on $\mathscr{A}^{\prime}$ defined by the condition that for any $A \in \mathscr{A}_{j}^{\prime}=\operatorname{End}\left(\mathscr{V}_{j}^{\prime}\right), j>0$, it is a vector state corresponding to $w^{*} \otimes w$ which is $G$-invariant. Consider now the state $\psi \otimes \psi^{\prime}$ on $\mathscr{A} \otimes \mathscr{A}^{\prime}$. As $G$ acts trivially on the vector state in $\mathscr{A}_{j}^{\prime}$ for all $j$, the composite state belongs to the same $G$-invariant stable phase as $(\psi, \mathscr{A})$.

After re-writing the algebra of observables as

$$
\operatorname{End}\left(\mathscr{W} \otimes \mathscr{W}^{*}\right) \otimes \operatorname{End}\left(\mathscr{W} \otimes \mathscr{W}^{*}\right) \otimes \ldots
$$

it is easy to see that the state $\psi \otimes \psi^{\prime}$ is related by a $G$-equivariant LGA to a factorized pure state which is in the trivial $G$-invariant phase. Indeed, since $\mathscr{W}$ contains a $G$-invariant vector $w^{\prime}, \mathscr{W} \otimes \mathscr{W}^{*}$ contains a $G$-invariant 2-plane spanned by vectors $w \otimes w^{*}$ and $w^{\prime} \otimes w^{\prime *}$. Let $U$ be a unitary operator on $\mathscr{W} \otimes \mathscr{W}^{*}$ which acts by identity on the orthogonal complement of this plane and rotates $w \otimes w^{*}$ into $w^{\prime} \otimes w^{\prime *}$. Consider a local unitary circuit on $\mathscr{A} \otimes \mathscr{A}^{\prime}$ which acts on $\mathscr{A} \otimes \mathscr{A}^{\prime}$ by conjugation with $U \otimes U \otimes U \otimes \ldots$. It maps $\psi \otimes \psi^{\prime}$ to a factorized pure state on $\mathscr{A} \otimes \mathscr{A}^{\prime}$ with $v_{0}=w^{\prime}$ and $v_{j}=w^{\prime *} \otimes w^{\prime}$ for $j>0$. All these vectors are $G$-invariant. Thus $\psi$ is in the trivial $G$-invariant stable phase 6

In general, we can write the algebra $\mathscr{A}$ as a tensor product of sub-algebras $\mathscr{A} \geq 0$ and $\mathscr{A}<0$ corresponding to $j \geq 0$ and $j<0$. Since the state $\psi$ on $\mathscr{A}$ is assumed factorized, it is sufficient to consider the restriction of $\psi$ to one of these sub-algebras, say $\mathscr{A}_{\geq 0}$. Then we apply the argument of the above paragraph to each of the factors $\mathscr{A}_{j}$ separately. Note that the auxiliary system $\mathscr{A}^{\prime}$ in this case has $\log d_{j}^{\prime}$ growing with $j$ even if the dimension $d_{j}$ of $\mathscr{A}_{j}$ is bounded. However, it is easy to see that if $\log d_{j}$ grows at most as a power of $j$, then so does $\log d_{j}^{\prime}$. Thus it is still true that $\psi$ is in the trivial $G$-invariant stable phase.

\section{B Local computability of the index}

An important property of the index is its local computability, i.e. that one can compute $\nu(g, h)$ up to $O\left(L^{-\infty}\right)$ accuracy while having an access only to a disk of

\footnotetext{
${ }^{6}$ This argument is a version of the Eilenberg swindle.
} 
radius $L$. Let us fix a disk $\Gamma_{2 L}=[-2 L, 2 L]$, and let $R_{L}^{g}$ be a unitary $\prod_{j \in[-L, L]} R_{j}(g)$. We assume that $\Psi$ can be produced from $\Omega$ by some LGA $\alpha_{F}$ with $f$-local $F$ for some MDP function $f(r)=O\left(r^{-\infty}\right)$.

First, note that by Proposition 1 and Lemma 4.1 we have

$$
U_{>j}^{g}|\Psi\rangle=\Pi\left(\mathcal{V}_{>j}^{g}\right)|\Psi\rangle .
$$

for some observable $\mathcal{V}_{>j}^{g}$ which is $g$-localized at $j$ for some function $g$ that depends on $f$ only. Therefore, we can find local on $\Gamma_{2 L}$ observables $\mathcal{V}_{L}^{g}$ and $\mathcal{V}_{-L}^{g}$ which are $g(r)$-localized at $j=L$ and $j=(-L)$, correspondingly, such that

$$
\Pi\left(R_{L}^{g}\right)|\Psi\rangle=\Pi\left(\mathcal{V}_{L}^{g}\right) \Pi\left(\mathcal{V}_{R}^{g}\right)|\Psi\rangle+O\left(L^{-\infty}\right) .
$$

The index can be computed as

$$
\begin{gathered}
\nu(g, h)=\left\langle\Psi\left|\left(U_{>j}^{g h}\right)^{-1} U_{>j}^{g} U_{>j}^{h}\right| \Psi\right\rangle=\left\langle\Psi\left|\Pi\left(\mathcal{V}_{>j}^{g h}\right)^{-1} U_{>j}^{g} \Pi\left(\mathcal{V}_{>j}^{h}\right)\right| \Psi\right\rangle= \\
=\left\langle\Psi\left|\Pi\left(\mathcal{V}_{>j}^{g h}\right)^{-1} \Pi\left(\rho_{>j}^{g}\left(\mathcal{V}_{>j}^{h}\right)\right) \Pi\left(\mathcal{V}_{>j}^{g}\right)\right| \Psi\right\rangle= \\
=\Psi\left(\left(\mathcal{V}_{>j}^{g h}\right)^{-1} \rho_{>j}^{g}\left(\mathcal{V}_{>j}^{h}\right) \mathcal{V}_{>j}^{g}\right)
\end{gathered}
$$

Therefore

$$
\nu(g, h)=\Psi\left(\left(\mathcal{V}_{L}^{g h}\right)^{-1} R_{L}^{g} \mathcal{V}_{L}^{h}\left(R_{L}^{g}\right)^{-1} \mathcal{V}_{L}^{g}\right)+O\left(L^{-\infty}\right) .
$$

Note that the $O\left(L^{-\infty}\right)$ term depends on $f(r)$ only, and by taking $L$ large enough we can compute the index with any given accuracy. Therefore if we have an interpolating $G$-invariant invertible state $\psi$ which is $f$-close to a $G$-invariant invertible state $\psi_{1}$ on the left half-chain and $f$-close to a $G$-invariant invertible state $\psi_{2}$ on the right halfchain, then the indices of $\psi_{1}$ and $\psi_{2}$ must be the same. In particular, a non-trivial index for $\psi_{1}$ is an obstruction for the existence of such an interpolation between $\psi_{1}$ and a pure factorized state $\psi_{2}$.

\section{References}

[1] Lukasz Fidkowski and Alexei Kitaev. "Topological phases of fermions in one dimension". In: Physical review B 83.7 (2011), p. 075103.

[2] Xie Chen, Zheng-Cheng Gu, and Xiao-Gang Wen. "Classification of gapped symmetric phases in one-dimensional spin systems". In: Physical review b 83.3 (2011), p. 035107.

[3] Yoshiko Ogata. A classification of pure states on quantum spin chains satisfying the split property with on-site finite group symmetries. 2019. arXiv: 1908.08621 [math.0A].

[4] Yoshiko Ogata. "A Z $\mathrm{Z}_{2}$-Index of Symmetry Protected Topological Phases with Time Reversal Symmetry for Quantum Spin Chains". In: Communications in Mathematical Physics 374.2 (July 2019), pp. 705-734. DOI: 10.1007/s00220-019-03521-5. 
[5] Chris Bourne and Yoshiko Ogata. The classification of symmetry protected topological phases of one-dimensional fermion systems. 2020. arXiv: 2006. 15232 [math-ph].

[6] Taku Matsui. "Boundedness of entanglement entropy and split property of quantum spin chains". In: Reviews in Mathematical Physics 25.09 (2013), p. 1350017.

[7] Matthew B Hastings. "An area law for one-dimensional quantum systems". In: Journal of Statistical Mechanics: Theory and Experiment 2007.08 (2007), P08024.

[8] Alvin Moon and Yoshiko Ogata. "Automorphic equivalence within gapped phases in the bulk". In: Journal of Functional Analysis 278.8 (2020), p. 108422.

[9] Bei Zeng, Xie Chen, Duan-Lu Zhou, and Xiao-Gang Wen. Quantum information meets quantum matter: From quantum entanglement to topological phases of many-body systems. Quantum Science and Technology. Springer, New York, 2019.

[10] Matthew B Hastings and Xiao-Gang Wen. "Quasiadiabatic continuation of quantum states: The stability of topological ground-state degeneracy and emergent gauge invariance". In: Physical review b 72.4 (2005), p. 045141.

[11] Anton Kapustin and Nikita Sopenko. "Hall conductance and the statistics of flux insertions in gapped interacting lattice systems". In: Journal of Mathematical Physics 61.10 (2020), p. 101901.

[12] Alexei Kitaev. "On the classificaton of Short-Range Entangled states". Talk at Simons Center for Geometry and Physics, June 2013. URL: http://scgp.stonybrook.edu/archives/7874.

[13] Ola Bratteli and Derek W. Robinson. Operator algebras and quantum statistical mechanics. 2. Equilibrium states. Models in quantum statistical mechanics. 2nd ed. Texts and Monographs in Physics. Springer-Verlag, Berlin, 1997.

[14] Ola Bratteli and Derek W. Robinson. Operator algebras and quantum statistical mechanics. 1. $C^{*}$ - and $W^{*}$-algebras, symmetry groups, decomposition of states. 2nd ed. Texts and Monographs in Physics. Springer-Verlag, New York, 1987.

[15] Chris Bourne and Hermann Schulz-Baldes. "On Z2-indices for ground states of fermionic chains". In: (2019).

[16] M. Fannes. "A continuity property of the entropy density for spin lattice systems". In: Comm. Math. Phys. 31 (1973), pp. 291-294. 
[17] Barry Simon. Trace ideals and their applications. Second. Vol. 120. Mathematical Surveys and Monographs. American Mathematical Society, Providence, RI, 2005, pp. viii+150. ISBN: 0-8218-3581-5.

[18] Yichen Huang. "Area law in one dimension: Degenerate ground states and Renyi entanglement entropy". In: arXiv preprint arXiv:1403.0327 (2014).

[19] Theodor Bröcker and Tammo Tom Dieck. Representations of compact Lie groups. Vol. 98. Springer Science \& Business Media, 2013. 